\title{
Opinion Dynamics in Social Networks with Hostile Camps: Consensus vs. Polarization
}

\author{
Anton V. Proskurnikov, Alexey Matveev and Ming Cao
}

\begin{abstract}
Most of the distributed protocols for multi-agent consensus assume that the agents are mutually cooperative and "trustful", and so the couplings among the agents bring the values of their states closer. Opinion dynamics in social groups, however, require beyond these conventional models due to ubiquitous competition and distrust between some pairs of agents, which are usually characterized by repulsive couplings and may lead to clustering of the opinions. A simple yet insightful model of opinion dynamics with both attractive and repulsive couplings was proposed recently by $C$. Altafini, who examined first-order consensus algorithms over static signed graphs. This protocol establishes modulus consensus, where the opinions become the same in modulus but may differ in signs. In this paper, we extend the modulus consensus model to the case where the network topology is an arbitrary time-varying signed graph and prove reaching modulus consensus under mild sufficient conditions of uniform connectivity of the graph. For cut-balanced graphs, not only sufficient, but also necessary conditions for modulus consensus are given.
\end{abstract}

Index Terms-Opinion dynamics, consensus, clustering, agents

\section{INTRODUCTION}

For multi-agent networks, the striking phenomenon of global consensus caused by only local interactions has attracted long-standing interest from the research community. The interest is motivated by numerous natural phenomena and engineering designs related to reaching synchrony or agreement among the agents. Examples include, but not limited to, intelligence of large biological populations and multi-robot teams. We refer the reader to [1]-[3] for excellent surveys of recent research on consensus protocols and their applications, as well as historical milestones.

Starting from the DeGroot algorithm of "iterative pooling" [4] for distributed decision making, many consensus algorithms were based on the principle of contraction: every

The work was supported in part by the European Research Council (ERCStG- 307207), St. Petersburg State University, grant 6.38.230.2015 and RFBR, grants 13-08-01014 and 14-08-01015. Lemma 13 was obtained by A.S. Matveev under sole support of Russian Science Fund (RSF) grant 1421-00041 at St. Petersburg State University. Theorems 5 and 6 were obtained by A.V. Proskurnikov under sole support of RSF grant 14-29-00142 at IPME RAS. The results were partly reported on IEEE Multiconference on Systems and Control MSC 2014 (Antibes, France) and IEEE Conference on Decision and Control CDC 2014 (Los Angeles, CA, USA).

A.V. Proskurnikov is with ENTEG, Faculty of Mathematics and Natural Sciences, University of Groningen, Groningen, the Netherlands and also with St. Petersburg State University, ITMO University and Institute for Problems of Mechanical Engineering of Russian Academy of Sciences (IPME RAS), St.Petersburg, Russia; avp1982@gmail . com

A. Matveev is with St. Petersburg State University, St. Petersburg, Russia; almat 1712 @yahoo.com

M. Cao is with ENTEG, Faculty of Mathematics and Natural Sciences, University of Groningen, Groningen, The Netherlands; m. cao@rug.n I agent's state constantly evolves to the relative interior of the convex hull spanned by its own and neighbors' states. Hence, the convex hull spanned by the states of the agents, driven by such a protocol, is shrinking over time. Based on the Lyapunov-like properties of this convex hull [5], [6] or relevant results on convergence of infinite products of stochastic matrices [1], [2], [7], stability properties of contracting iterations were examined intensively with special attention on the effect of time-varying interaction topologies. Necessary and sufficient conditions for consensus under bidirectional [5], [8], [9] and cut-balanced graphs [10] boil down to repeated joint connectivity of the network. For general directed graphs the sufficient condition of uniform quasi-strong connectivity (UQSC) [6], is considered to be "the weakest assumption on the graph connectivity such that consensus is guaranteed for arbitrary initial conditions" [11]. This common belief has recently been confirmed by results in [12], [13] stating that the UQSC is necessary and sufficient for robust consensus and consensus with exponential convergence. Many high-order consensus algorithms either extend their first-order counterparts [1], [3] or are squarely based on them [14].

Unlike teams of agents that achieve a common goal due to cooperation, networks where agents can both cooperate and compete (sometimes referred to as coopetitive networks [15], [16]) still demand more thorough mathematically rigorous analysis. In social networks, competition, antagonism and distrust between social actors and their groups are ubiquitous [17], [18], which are usually modeled by repulsive couplings or negative ties [19] among the agents. A specific example of such couplings observed in dyadic interactions, is reactance [20] which leads to boomerang effects, first described in [21]: in the process of persuasion, opinions (even close to each other initially) can become opposite. Analogous phenomenon, referred to as the group polarization [22], has long been studied in social psychology: the community divides into two groups, each reaching consensus; the consensus opinions are not only opposite, but often further away from each other than the two initial average opinions of the corresponding groups. Analysis of real-world social networks (e.g. users of social web-sites [17]) shows the strong correlation between polarization and structural balance [17], [18] of positive and negative ties. The latter property implies that community splits into two hostile camps (e.g. votaries of two political parties), where the relations inside each faction are cooperative.

It is known that agents' repulsion can lead to the clustering behavior in a complex network [23]. The possibility of clustering in social groups due to negative ties was demonstrated in [19] (see also references therein); these effects are still waiting 
for mathematically rigorous analysis. Most of the existing works on opinion dynamics focus on the persistent disagreement and clustering of opinions caused by bounded confidence [24], [25] or, more generally, biased assimilation [26]: agents readily adopt opinions of like-minded neighbors, accepting the "deviating" opinions with discretion. In [27], [28] Altafini proposed a simple yet instructive mathematical model of opinion polarization over structurally balanced graphs, extending conventional first-order consensus algorithms to the case with antagonistic interactions. These protocols were examined under the assumption that the interaction graph is static and strongly connected and shown to establish modulus consensus [29], where the opinions agree in modulus but may differ in signs. If the graph is structurally balanced, the modulus of the final opinion is generally non-zero and opinions either reach consensus or polarize ("bipartite consensus" [28] is established); otherwise, opinions converge to zero.

Mathematical examination of polarization behavior due to antagonism is among the first important steps towards understanding the dynamics of networks consisting of both cooperative and competitive agents. Such networks are not confined to social systems; repulsive interactions play an important role in e.g. motion control of swarms and other multi-agent formations, where agents may avoid collision [30], [31] and distribute evenly on circular or other closed curves [32]-[34]. So a number of papers have been published recently studying this class of networks [15], [35]-[39].

The aforementioned papers, however, mainly focus on the case where the interaction topology is static. In the present paper, we consider Altafini's model on opinion dynamics over general directed time-varying graphs. Removing the restrictions of static topologies not only allows one to analyze dynamics of real social networks, where the agents may change their relationships from friendship to hostility and vice versa, but also enables one to extend the result to nonlinear protocols. In fact we will examine nonlinear algorithms in the common framework as linear ones, getting rid of the restrictions such as monotonicity [27], [28], [40].

Our main result states that modulus consensus is established if the topology is uniformly strongly connected. Unlike cooperative networks, the uniform strong connectivity cannot be relaxed to the uniform quasi-strong connectivity, which is a commonly adopted condition for consensus over directed time-varying graph [11], [41]. At the same time, the condition of uniform strong connectivity is in general not necessary, and filling the gap between necessary and sufficient conditions remains a tough problem even in the cooperative case. However, we fill this gap in the special case of cutbalanced graphs, extending necessary and sufficient consensus criterion from [10] to modulus consensus over signed graphs. It should be noticed that results from [10] are not directly applicable to signed graphs; in the special case of bidirectional or "reciprocal interactions" they were extended to the signed case by the lifting technique [37]. We will make further remarks on this in the corresponding sections. The results were partly reported in our conference papers [42], [43].

The paper is organized as follows. Section [II introduces some preliminary concepts and notations. Section [II] gives the setup of the problem in question. Section $[\mathrm{IV}$ presents the main results. Section VI offers the proof of the main results.

\section{Preliminaries}

Throughout the paper $m: n$, where $m, n$ are integers and $m \leq n$, stands for the sequence $\{m, m+1, \ldots, n\}$. The sign of a number $x \in \mathbb{R}$ is denoted by $\operatorname{sgn} x \in\{-1,0,1\}$. The abbreviation "a.a." stands for "almost all" (except for the set of zero Lebesgue measure). Given a matrix $L=\left(l_{j k}\right)$, let abs $L \triangleq\left(\left|l_{j k}\right|\right)$. We also introduce the matrix norm $|L|_{\infty} \triangleq$ $\max _{j} \sum_{k}\left|L_{j k}\right|$. As usual, for a column vector $x \in \mathbb{R}^{N}$, one has $|x|_{\infty}=\max _{j}\left|x_{j}\right|$ and it is easily shown that $|L|_{\infty}=$ $\sup \frac{|L x|_{\infty}}{|x|_{\infty}}$, where the supremum is over all column vectors $x \neq 0$ of appropriate dimensions. Let $\overline{1}_{N} \triangleq(1,1, \ldots, 1)^{T} \in$ $\mathbb{R}^{N}$. Given $x \in \mathbb{R}$, let $x^{+}=\max (x, 0)$ and $x^{-}=(-x)^{+}$, hence $x=x^{+}-x^{-}$and $|x|=x^{+}+x^{-}$.

\section{A. Signed graphs and their properties}

A (weighted directed) signed graph is a triple $G=$ $(V, E, A)$, where $V=\left\{v_{1}, \ldots, v_{N}\right\}$ stands for the set of nodes, $E \subset V \times V$ is a set of arcs and $A=\left(a_{j k}\right) \in \mathbb{R}^{N \times N}$ is a signed adjacency matrix, i.e. $a_{j k} \neq 0$ if and only if $\left(v_{k}, v_{j}\right) \in E$. Throughout the paper, we confine ourselves to graphs that have no self-loops $\left(a_{j j}=0 \forall j\right)$ and are digon sign-symmetric [28], i.e. any pair of opposite arcs (if exists) is identically signed: $a_{j k} a_{k j} \geq 0 \forall j, k$. Identifying the set of nodes $V$ with 1 : $N$, there is a one-to-one correspondence between signed graphs and their adjacency matrices $A \in \mathbb{R}^{N \times N} \mapsto G[A]=(1: N, E[A], A)$, where $E[A]=\left\{(j, k): a_{k j} \neq 0\right\}$.

Given $\varepsilon>0$, let $A^{\varepsilon}=\left(a_{j k}^{\varepsilon}\right)$ stand for the "truncated" adjacency matrix: $a_{i j}^{\varepsilon}=a_{i j}$ when $\left|a_{i j}\right| \geq \varepsilon$ and $a_{i j}^{\varepsilon}=0$ otherwise. The corresponding graph $G^{\varepsilon} \triangleq G\left[A^{\varepsilon}\right]$ is obtained from $G=G[A]$ by removing arcs of absolute weight less than $\varepsilon$ and we call it $\varepsilon$-skeleton of the graph $G$.

A path connecting nodes $v$ and $v^{\prime}$ is a sequence of nodes $v_{i_{0}}:=v, v_{i_{1}}, \ldots, v_{i_{n-1}}, v_{i_{n}}:=v^{\prime}(n \geq 1)$ such that $\left(v_{i_{k-1}}, v_{i_{k}}\right) \in E$ for $k \in 1: n$. A path where $v_{i_{0}}=v_{i_{n}}$ is referred to as a cycle. The cycle is positive if $a_{i_{0} i_{1}} a_{i_{1} i_{2}} \ldots a_{i_{n-1} i_{n}}>0$ and negative otherwise. The digonsymmetric strongly connected graph is structurally balanced if and only if all its oriented cycles are positive [17], [28]. A node is called root if it can be connected with a route to any other node of the graph. A graph is strongly connected (SC) if a path between any two different nodes exists. The graph is quasi-strongly connected (QSC) if it has at least one root. Any SC graph is also QSC, each node being a root. A graph whose $\varepsilon$-skeleton is SC (respectively, QSC) is called strongly $\varepsilon$-connected (respectively, quasi-strongly $\varepsilon$-connected).

Given a graph $G=(V, E, A)$, its subgraph is a graph $G^{\prime}=$ $\left(V^{\prime}, E^{\prime}, A^{\prime}\right)$, where $V^{\prime} \subseteq V, E^{\prime} \subseteq\left(V^{\prime} \times V^{\prime}\right) \cap E$ and $A^{\prime}=$ $\left(a_{i j}\right)_{i, j \in V^{\prime}}$ stands for the corresponding submatrix of $A$. We call a subgraph in-isolated if no arc comes from $V \backslash V^{\prime}$ to $V^{\prime}$, i.e. $a_{j i}=0 \forall i \in V^{\prime}, j \notin V^{\prime}$.

We call two disjoint non-empty sets $V_{1}, V_{2} \subseteq V$ hostile camps in the graph $G$ if $a_{j k} \geq 0$ when $j, k \in V_{1}$ or $j, k \in V_{2}$ 
and $a_{j k} \leq 0$ whenever $j \in V_{1}, k \in V_{2}$ or $j \in V_{2}, k \in V_{1}$. The graph is structurally balanced (SB) [17], [28] if the set of its nodes can be divided into two hostile camps $V=V_{1} \cup V_{2}$. The digon-symmetric SC graph is structurally balanced if and only if any cycle in it is positive [17], [28].

Following [28], we define the Laplacian matrix $L=L[A]$ of the signed graph $G[A]$ as follows

$$
L[A] \triangleq\left(L_{j k}\right)_{j, k=1}^{N}, \quad L_{j k}:=\left\{\begin{array}{l}
-a_{j k}, j \neq k \\
\sum_{m=1}^{N}\left|a_{j m}\right|, j=k .
\end{array}\right.
$$

Equation (1) is a straightforward extension of the conventional definition of the Laplacian matrix of a weighted graph [44] to the case of signed weights. As implied by the Gershgorin disk theorem [28], $L[A]$ has no eigenvalues in the closed left half-plane $\overline{\mathbb{C}}_{-}=\{\lambda \in \mathbb{C}: \operatorname{Re} \lambda \leq 0\}$ except for possibly $\lambda=0$. Unlike the unsigned case, in general $L[A]$ may have no zero eigenvalue and hence $-L[A]$ may be a Hurwitz matrix. For a SC graph $G=G[A]$ this is the case if and only if $G$ is not structurally balanced [28, Lemma 2].

\section{B. Some important types of time-varying signed graphs}

Throughout the paper, the term time-varying (signed) graph means the graph $G[A(t)]$, where a time-dependent matrix $A(t) \in \mathbb{R}^{N \times N}$ is Lebesgue measurable and locally bounded. Given such a graph $G(t)=G[A(t)]$, we say a node $j$ is essentially connected to a node $k$ if $\int_{t_{0}}^{\infty}\left|a_{j k}(s)\right| d s=\infty$ for some $t_{0} \geq 0$ (the latter inequality then holds for any $t_{0} \geq 0$ since $a_{j k}$ is locally bounded). Let $\mathcal{E}=\mathcal{E}[A(\cdot)]$ stand for the set of all such pairs $(j, k)$. Following [9], we call the graph $\mathcal{G}[A(\cdot)]=(1: N, \mathcal{E})$ the graph of essential interactions and say that the graph $G(\cdot)$ is essentially strongly connected (ESC) if $\mathcal{G}[A(\cdot)]$ is strongly connected. Likewise, $G(\cdot)$ is essentially quasi-strongly connected (EQSC) if $\mathcal{G}[A(\cdot)]$ is QSC.

The graph $G[A(\cdot)]$ is said to be uniformly strongly connected (USC) if there exist constants $T>0$ and $\varepsilon>0$ such that the graph $G\left[\int_{t}^{t+T} a b s A(s) d s\right]$ is strongly $\varepsilon$-connected for any $t \geq 0$. By replacing the word "strongly" in the latter definition with "quasi-strongly", one defines uniformly quasi-strongly connected (UQSC) time-varying graph. It may be easily shown that the USC (respectively UQSC) graph is always ESC (respectively, EQSC), while the inverse is not valid.

The graph $G[A(\cdot)]$ is cut-balanced [10] if a constant $K \geq 1$ exists such that for any partition of the nodes $V^{\prime} \cup V^{\prime \prime}=1: N$, $V^{\prime} \cap V^{\prime \prime}=\emptyset$, the following inequalities hold

$$
K^{-1} \sum_{j \in V^{\prime}} \sum_{k \in V^{\prime \prime}}\left|a_{k j}\right| \leq \sum_{j \in V^{\prime}} \sum_{k \in V^{\prime \prime}}\left|a_{j k}\right| \leq K \sum_{j \in V^{\prime}} \sum_{k \in V^{\prime \prime}}\left|a_{k j}\right| \text {. }
$$

A typical example of a cut-balanced graph is the typesymmetric graph [10], which means the existence of $K \geq 1$ such that

$$
K^{-1}\left|a_{k j}(t)\right| \leq\left|a_{j k}(t)\right| \leq K\left|a_{k j}(t)\right| \forall t \geq 0 \forall j \neq k .
$$

Other examples include weight-balanced graphs, see [10] for details. As implied by [10, Lemma 1], for cut-balanced graphs, the EQSC property implies ESC; precisely, any quasi-strongly connected component of the digraph $\mathcal{G} \triangleq \mathcal{G}[A(\cdot)]$ is strongly connected, and a path between $j$ and $k$ exists if and only if the path from $k$ to $j$ exists.

\section{Problem SetuP}

Consider a group of $N \geq 2$ agents indexed 1 through $N$, the opinion of the $i$ th agent is denoted by $x_{i} \in \mathbb{R}$ and we define $x:=\left(x_{1}, \ldots, x_{N}\right)^{T} \in \mathbb{R}^{N}$. The agents update their opinions in accordance with a distributed protocol as follows:

$$
\dot{x}(t)=-L[A(t)] x(t), t \geq 0,
$$

which can be written componentwise as

$$
\dot{x}_{j}(t)=\sum_{k=1}^{N}\left|a_{j k}(t)\right|\left(x_{k}(t) \operatorname{sgn} a_{j k}(t)-x_{j}(t)\right) \forall j .
$$

Here $A(t)=\left(a_{j k}(t)\right)$ is a locally bounded matrix-valued function which describes the interaction topology of the network and $a_{j j}(t) \equiv 0$. At time $t \geq 0$, the opinion of the $j$ th agent is influenced by agents for which $a_{j k} \neq 0$ ("neighbors"). Unlike conventional consensus protocols [44] this influence may be either cooperative (when $a_{j k}>0$ ) or competitive (when $a_{j k}<0$ ). The coupling term $\left|a_{j k}\right|\left(x_{k}\right.$ sgn $\left.a_{j k}-x_{j}\right)$ in (5) drives the opinion of the $j$ th agent, respectively, either towards the opinion of the $k$ th one or against it.

In [28] protocol (4) has been carefully examined, assuming the interaction graph is constant $(A(t) \equiv A)$ and strongly connected. It was shown that the steady-state opinions always agree in modulus, but generally differ in signs; in other words, the modulus consensus of opinions [29] is established.

Definition 1: The protocol (4) establishes modulus consensus, if for any $x(0)$ a number $x_{*} \geq 0$ exists such that

$$
\lim _{t \rightarrow+\infty}\left|x_{i}(t)\right|=x_{*} .
$$

The following lemma shows that there are two essentially different types of modulus consensus: "trivial" with $x_{*}=0$ for all $x(0)$ (the system (4) is asymptotically stable) and "nontrivial", where $x_{*} \neq 0$ for a.a. $x(0)$.

Lemma 1: Suppose that protocol (4) establishes modulus consensus. Then there exist vectors $v, \rho \in \mathbb{R}^{N}$ with $\rho_{1}, \ldots, \rho_{N}= \pm 1$ such that for any solution of (4) one has

$$
\lim _{t \rightarrow+\infty} x(t)=\rho v^{T} x(0) \Leftrightarrow \lim _{t \rightarrow+\infty} x_{j}(t)=\rho_{j} v^{T} x(0) .
$$

Lemma 1 shows that in the "non-trivial" case $v \neq 0$, opinions either reach consensus $\left(\rho_{1}=\ldots=\rho_{N}\right)$ or polarize $\left(\rho_{i}\right.$ have different signs) whenever $v^{T} x(0) \neq 0$. For both situations we say that the protocol establishes bipartite consensus.

Definition 2: We call the protocol (4) stabilizing, if $\lim _{t \rightarrow \infty} x_{j}(t)=0 \forall j \forall x(0)$. The protocol establishes bipartite consensus if (7) holds with some $v \neq 0$; it establishes consensus if additionally $\rho=\overline{1}_{N}$ or $\rho=-\overline{1}_{N}$.

It was proved in [28] if $A(t) \equiv A$, then the protocol is stabilizing (that is, $-L[A]$ is a Hurwitz matrix) unless the graph $G[A]$ is structurally balanced (SB). The latter property implies that a community is divided into two hostile camps (such as votaries of two political parties), where each agent cooperates with its camp-mates, competing with agents from 
the opposite camp. A special case of SB graphs is the graph with non-negative weights $a_{j k} \geq 0$ where one of the camps is empty. In this case strong connectivity (SC) and even quasistrong connectivity (QSC) imply consensus [1]. The case of general SB and SC graph is reducible to this case by means of the gauge transformation [28], which allows to prove (6), where $x_{*}$ depends on the initial conditions. If both hostile camps are not empty, the opinions polarize. In other words, structural balance implies bipartite consensus. A generalization of the gauge transformation from [28] is the lifting approach from [37], splitting each agent into a pair of virtual agents with opposite opinions, after which the original dynamics can be considered as a projection of some larger network with purely cooperative interactions. This approach can be applied also to some time-varying networks.

In Section IV-A we further refine Altafini's results on modulus consensus over static graphs by discarding the strong connectivity assumption. We show that for structurally balanced graphs modulus consensus is established if and only if the graph is QSC; in fact, in this case bipartite consensus is established. Conversely, bipartite consensus is reached only when the graph is structurally balanced and QSC. If the graph has no structurally balanced in-isolated subgraphs, the protocol is stabilizing. Thus we offer necessary and sufficient conditions for modulus consensus for general static graph.

The main concern of this paper is modulus consensus over time-varying signed graphs. In [28] this problem was considered only for the very special case where the graph is constantly strongly connected, has time-invariant signs of the arcs and also weight-balanced (this assumption was not explicitly mentioned, but in fact was used in the proof which appeals to [44, Theorem 9]). Below we relax these restrictions. Dealing with real-world social networks, the time-invariance of such relationships between individuals as friendship and hostility is evidently a non-realistic assumption. What is more important, the opinion dynamics in social networks are usually considered to be nonlinear [19], [24]. Such models are often reducible to the linear case by introducing time-varying gains, depending on the solution; however, the corresponding graphs can hardly be weight-balanced. Our techniques allow us to examine both linear and nonlinear consensus protocols from [27], [28] in the common framework. Although it is a hard problem to find explicitly the ultimate opinion vector in the case of time-varying topologies, Lemma 1 shows that there are similarities with the static case.

A common techniques used to prove consensus in the case of cooperative agents is the shrinking property of the convex hull, spanned by agent's opinions. Under the UQSC property of the graph, the diameter of this convex hull may serve as a Lyapunov function [5], [6], [11]. The UQSC condition is not necessary in general [5], considered as "the weakest assumption on the graph connectivity such that consensus is guaranteed for arbitrary initial conditions" [11], and becomes necessary under additional restriction of uniform convergence [6]. On the other hand, the EQSC condition is always necessary for consensus yet insufficient in the case of directed topologies [5]. This gap between necessary and sufficient conditions has been filled recently for type-symmetric and other cut-balanced graphs [9], [10] where EQSC is not only necessary but also sufficient for consensus.

Under antagonistic interactions between the agents, the convex hull spanned by opinions is not shrinking, and the only available Lyapunov function is the maximal modulus, which will be shown to be non-increasing and thus converging to a limit. However, the UQSC property in general does not guarantee that the minimal modulus also converges to the same limit (as will be shown by a counter example). To provide this, one requires stronger USC conditions. Whereas EQSC property is necessary for bipartite consensus, it is not necessary for stability, as illustrated by the following trivial example. Let $A=\operatorname{diag}\left(A_{1}, A_{2}\right)$, where both graphs $G\left[A_{1}\right]$ and $G\left[A_{2}\right]$ are strongly connected and structurally unbalanced. As follows from [28], the matrices $\left(-L\left[A_{1}\right]\right)$ and $\left(-L\left[A_{2}\right]\right)$ are Hurwitz, which also holds for $(-L[A])=-\operatorname{diag}\left(L_{1}[A], L_{2}[A]\right)$ and thus the protocol is stabilizing. The static graph $G[A]$ is not QSC and thus not EQSC. Filling this gap between the necessary and sufficient conditions for modulus consensus that is even "wider" than in the cooperative case, is a tough open problem. However, adopting the techniques from [9], we fill this gap for cut-balanced graphs by offering necessary and sufficient conditions of modulus consensus (Subsection IV-C).

\section{Main Results}

This section is organized as follows. We start with modulus consensus criteria for static graphs which extend results from [28] by discarding the assumption of strong connectivity in them (Subsection IV-A. We show that the necessary and sufficient condition for bipartite consensus is structural balance and QSC, and give also necessary and sufficient conditions for stability. The next Subsection IV-B deals with the case of general time-varying graphs. We show sufficiency of the USC condition for modulus consensus and demonstrate that, unlike the cooperative case, this condition cannot be relaxed to UQSC. In the last Subsection IV-C we focus on modulus consensus over cut-balanced graphs. In this case it is possible to give necessary and sufficient conditions for both types of modulus consensus, whereas filling the gap between necessary and sufficient conditions for modulus consensus under general directed graphs remains a tough open question.

\section{A. Time-invariant protocols}

Throughout this section $A(t) \equiv A$ is a constant matrix. We start with the case of structurally balanced graph. In this case, a gauge transformation [28] exists which reduces the protocol to a cooperative one, whose properties are well established.

Lemma 2: Let $G[A]$ be structurally balanced. Then $L[A]$ has eigenvalue at 0 and the following claims are equivalent:

1) the graph $G[A]$ is QSC;

2) the linear subspace $\operatorname{ker} L[A] \subset \mathbb{R}^{n}$ has dimension 1 ; 3) the protocol (4) establishes modulus consensus.

If these claims hold, then $\rho, v$ from Lemma 1 are respectively the right and the left eigenvectors of $L[A]$ at 0 , hence $v^{T} L[A]=L[A] \rho=0$ and $v \neq 0$, so protocol establishes bipartite consensus. If $A$ is a non-negative matrix (one of the 


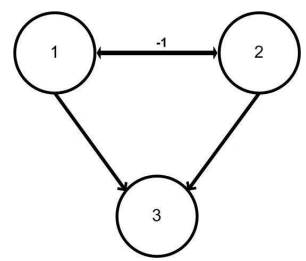

Fig. 1. Static QSC graph: modulus consensus is impossible if $a_{31} a_{32}>0$

hostile camps is empty), the protocol establishes consensus, and otherwise, opinions polarize.

In the case where $G[A]$ is structurally balanced yet not QSC (so modulus consensus is not reached), the structure of steadystate opinions may be described in terms of the maximum out-forest matrix as done in [45] for cooperative agents.

As follows from Lemma 2, a protocol with structurally balanced graph cannot be stabilizing. Therefore, stability is also impossible when the graph contains an in-isolated structurally balanced (ISB) subgraph, in other words, the group involves hostile camps whose members ignore the opinions of the remaining agents. The nodes of such a subgraph, if existed, would reach bipartite consensus of opinions independently of the remaining agents. The following theorem gives a criterion for modulus consensus over static graphs, showing that existence of ISB subgraphs is the only obstacle for stability.

Theorem 1: Let $A(t)=$ const. The protocol (4) is stabilizing if and only if the graph is neither SB itself nor contains an ISB subgraph. Bipartite consensus is established if and only if $G[A]$ is structurally balanced and QSC: if $a_{j k} \geq 0 \forall j, k$, then consensus is established; otherwise, opinions polarize.

Example 1. Consider a team of $N=3$ agents with states $x_{1}(t), x_{2}(t), x_{3}(t)$. Assume that $a_{12}=a_{21}=-1$ (see Fig. 1) and $a_{31} a_{32}>0$. Thus the equations are

$$
\begin{array}{r}
\dot{x}_{1}=\left(-x_{2}-x_{1}\right), \dot{x}_{2}=\left(-x_{1}-x_{2}\right), \\
\dot{x}_{3}=a_{31} x_{1}+a_{32} x_{2}-\left(\left|a_{31}\right|+\left|a_{32}\right|\right) x_{3},
\end{array}
$$

and the graph is not structurally balanced (agents 1 and 2 are constantly antagonistic, so the structural balance requires agent 3 to cooperate with only one of them, competing with the other, whereas in reality it cooperates with both agents 1 and 2). According to Theorem 11, modulus consensus is impossible (since the ISB subgraph with the set of nodes $\{1,2\}$ exists). This can also be shown in a straightforward way: the system has equilibria $(\xi,-\xi, \rho \xi)$, with $\rho \triangleq\left(a_{31}-\right.$ $\left.a_{32}\right) /\left(\left|a_{31}\right|+\left|a_{32}\right|\right) \in(-1 ; 1), \xi \in \mathbb{R}$.

This simple example illustrates that, unlike the cooperative case $\left(a_{j k} \geq 0\right)$, the protocol with static QSC graphs in general does not establish modulus consensus. To guarantee modulus consensus, one typically requires strong connectivity (assumed in [28]) or some other property, excluding ISB subgraphs.

\section{B. Protocols over dynamic signed graphs}

We start with the following useful lemma, which does not rely on any connectivity assumptions and shows, in particular, that solutions to (4) are always bounded.

Lemma 3: For any solution of system (4), the function $|x(t)|_{\infty}=\max _{i}\left|x_{i}(t)\right|$ is monotonically non-increasing: $|x(t)|_{\infty} \leq\left|x\left(t_{0}\right)\right|_{\infty}$ whenever $t \geq t_{0} \geq 0$. Equivalently, the Cauchy evolutionary matrix $\Phi\left(t ; t_{0}\right)$ of system (4) satisfies the inequality $\left|\Phi\left(t ; t_{0}\right)\right|_{\infty} \leq 1$ for $t \geq t_{0}$.

Lemma 3 implies, in particular, the existence of the limit $\lim _{t \rightarrow+\infty}|x(t)|_{\infty}$. The following theorem shows that under the uniform strong connectivity property and bounded coupling gains the modules of all opinions converge to the same limit.

Theorem 2: If $a_{j k}(t)$ are bounded and the graph $G[A(\cdot)]$ is USC, then the protocol (4) establishes modulus consensus.

As a corollary, we immediately obtain the well-known criterion for consensus under cooperative protocols.

Corollary 1: If $a_{j k}(t) \geq 0 \forall j, k$ for a.a. $t \geq 0$ and $G[A(\cdot)]$ is USC, then the protocol (4) establishes consensus.

It is well known that assumptions of Corollary 1 can be relaxed: in the case where $a_{j k} \geq 0$ the UQSC condition is sufficient for consensus [6], [11], [46]. Moreover, using the gauge transformation approach from [28], sufficiency of the UQSC property may be proved for a dynamic structurally balanced graph, provided that the subdivision into two "hostile camps" remains unchanged.

Lemma 4: Suppose that $V=1: N=V_{1} \cup V_{2}$, where $a_{j k}(t) \geq 0$ for any $t \geq 0$ if $j, k \in V_{1}$ or $j, k \in V_{2}$; otherwise, $a_{j k}(t) \leq 0$ for any $t \geq 0$. If the graph $G[A(\cdot)]$ is UQSC, the protocol establishes bipartite consensus (if $V_{1}=\emptyset$ or $V_{2}=\emptyset$ ) or bipartite consensus (when $V_{1}, V_{2} \neq \emptyset$ ).

Remark 1: Lemma 4 obviously remains valid if two hostile camps $V_{1}, V_{2}$ exist only for $t \geq t_{0}$, where $t_{0} \geq 0$. This observation makes the result of Lemma 4 applicable to topologies that evolve in order to achieve the structural balance in finite time (after which the signs of arcs remain unchanged); graph dynamics leading to structural balance in finite time were proposed in [47], [48].

However, in general the USC condition in Theorem 2 is not relaxable to UQSC. Example 1 in Subsection IV-A shows that even for static graphs the QSC property (equivalent to UQSC) does not guarantee modulus consensus unless the graph is structurally balanced. Our next example shows that the UQSC property is not sufficient neither when the graph remains structurally balanced but the relations of friendship and hostility between the agents evolve over time. We construct a protocol (4) with periodic piecewise-constant matrix $A(t)$, such that the graph $G[A(t)]$ is structurally balanced for any $t \geq 0$ and UQSC, and nevertheless modulus consensus is not established.

Example 2. Consider the more general system

$$
\begin{aligned}
& \dot{x}_{1}(t)=\left(-x_{2}(t)-x_{1}(t)\right), \dot{x}_{2}(t)=\left(-x_{1}(t)-x_{2}(t)\right), \\
& \dot{x}_{3}(t)=a_{31}(t)\left(x_{1}(t)-x_{3}(t)\right)+a_{32}(t)\left(x_{2}(t)-x_{3}(t)\right) .
\end{aligned}
$$

The functions $a_{31}, a_{32}$ are constructed as follows. Consider first system (8) with $a_{31}(t) \equiv 1, a_{32}(t) \equiv 0$ and the solution to (8) launched at the initial state $x_{1}(0)=1, x_{2}(0)=$ $-1, x_{3}(0)=-1 / 2$. It is evident that $x_{1}(t)=1=-x_{2}(t)$ for any $t \geq 0$ and $x_{3}(t) \uparrow 1$ as $t \rightarrow+\infty$. Therefore, there exists the first time instant $T_{0}>0$ such that $x_{3}\left(T_{0}\right)=1 / 2$. Notice that in the symmetric situation where $a_{31}(t) \equiv 0, a_{32}(t) \equiv 1$ and $x(t)$ is a solution to $(8)$ starting at $x_{1}(0)=1, x_{2}(0)=$ $-1, x_{3}(0)=1 / 2$, one has $x_{3}(t) \downarrow-1$ and $T_{0}$ is the first instant 
where $x_{3}\left(T_{0}\right)=-1 / 2$. Taking

$a_{31}(t)=1-a_{32}(t)=\left\{\begin{array}{l}1, t \in\left[0 ; T_{0}\right) \cup\left[2 T_{0} ; 3 T_{0}\right) \cup \ldots \\ 0, t \in\left[T_{0} ; 2 T_{0}\right) \cup\left[3 T_{0} ; 4 T_{0}\right) \cup \ldots\end{array}\right.$

one finally gets a $2 T_{0}$-periodic matrix $A(t)$, corresponding to the UQSC graph $G[A(\cdot)]$. Moreover, this graph is also quasi-strongly connected and structurally balanced at any time. Even so the solution to 8 starting at $x_{1}(0)=1, x_{2}(0)=$ $-1, x_{3}(0)=-1 / 2$ does not achieve modulus consensus. It can be easily shown that $x_{1}(t)=-x_{2}(t)=1$ for any $t \geq 0$. Since $a_{31}(t)=1$ and $a_{32}(t)=0$ when $t<T_{0}$, one has $x_{3}\left(T_{0}\right)=1 / 2$ by definition of $T_{0}$. On the next interval $t \in\left[T_{0} ; 2 T_{0}\right)$ one has $a_{31}(t)=0$ and $a_{32}(t)=1$ and hence $x_{3}\left(2 T_{0}\right)=-1 / 2$, so the solution $x(t)$ is periodic and $x_{3}(t) \in[-1 / 2 ; 1 / 2]$ whereas $\left|x_{1}(t)\right|=\left|x_{2}(t)\right|=1$.

Dealing with purely cooperative protocols, UQSC is considered to be not only sufficient for consensus but also "nearly" necessary. It may also be relaxed to EQSC for some types of graphs (e.g. cut-balanced ones). On the other hand, the EQSC condition is always necessary for consensus among cooperating agents, being in general not sufficient. As discussed in Section IV-A stability of (4) is possible without the EQSC property even for static graphs. However, EQSC is required for bipartite modulus consensus.

Lemma 5: If the protocol (4) establishes bipartite consensus, the graph $G[A(\cdot)]$ is EQSC.

Remark 2: In the general situation, where the graph is USC but the assumptions of Lemma 4 do not hold, it is difficult to distinguish between stability and bipartite consensus. A sufficient condition for stability was proved in [37]: the protocol is stabilizing, if for some $\varepsilon>0, T>0$ all the graphs $G\left[\int_{t}^{t+T} a b s A(s) d s\right]$ (where $t \geq 0$ ) have strongly connected and structurally unbalanced $\varepsilon$-skeletons [37]. This is the only case where the "lifted" network is proved to inherit the USC property [37]. Generally, this is not the case, so the approach [37] does not allow to derive Theorem 2 .

\section{Modulus consensus over cut-balanced graphs}

In the previous subsection, we get a sufficient condition for modulus consensus (the USC condition). In general, this property is not necessary for modulus consensus. Moreover, even the weaker EQSC condition is necessary for bipartite consensus but not for stability. Filling this gap between necessary and sufficient conditions is a hard open problem even for cooperative protocols. However, this gap has been recently filled for cooperative protocols with type-symmetric [9] and more general cut-balanced graphs [10]. The proofs from [10] are not applicable for signed graphs. However, adopting the approach from [9], we extend the result from [10] to the case of modulus consensus over signed graph, giving necessary and sufficient conditions for each type of modulus consensus.

Throughout this section the graph $G[A(\cdot)]$ is cut-balanced, i.e. the inequalities (2) hold for some $K \geq 1$. Recall the $j$ th agent essentially interacts with the $k$ th one if $\int_{0}^{\infty} a_{j k}(t) d t=$ $\infty$ and hence either $\int_{0}^{\infty} a_{j k}^{+}(t) d t=\infty$ or $\int_{0}^{\infty} a_{j k}^{-}(t) d t=$ $\infty$. We say agents essentially cooperate in the first case and essentially compete in the second situation (in general, both relations may hold). Let $\mathcal{E} \triangleq \mathcal{E}[A(\cdot)]$ and $\mathcal{E}^{+} \subseteq \mathcal{E}, \mathcal{E}^{-} \subseteq \mathcal{E}$ be the sets of those pairs of agents $(j, k)$ that respectively essentially cooperate and essentially compete.

If $\mathcal{E}^{+} \cap \mathcal{E}^{-}=\emptyset$, we assign the weights +1 and -1 to the arcs from $\mathcal{E}^{+}$and $\mathcal{E}^{-}$respectively, transforming $\mathcal{G} \triangleq \mathcal{G}[A(\cdot)]$ to a signed graph $\mathcal{G}^{ \pm}=\left(1: N, \mathcal{E},\left(s_{j k}\right)\right), s_{j k}=+1$ for $(j, k) \in \mathcal{E}^{+}$and $s_{j k}=-1$ for $(j, k) \in \mathcal{E}^{-}$.

As implied by [10 Lemma 1], for cut-balanced graphs the EQSC property implies ESC, precisely, any quasi-strongly connected component of the digraph $\mathcal{G} \triangleq \mathcal{G}[A(\cdot)]$ is strongly connected, and a path between $j$ and $k$ exists if and only if a path from $k$ to $j$ exists. From Lemma 5, the ESC condition is necessary for bipartite consensus. In the case of cooperative agents, this property is also sufficient for consensus [10]. However, in the case of signed graph, ESC is not sufficient without the "essential" structural balance.

Theorem 3: Assume the graph $G[A(\cdot)]$ is cut-balanced. The protocol (4) establishes bipartite modulus consensus if and only if $\mathcal{G}^{ \pm}$is well-defined $\left(\mathcal{E}^{+} \cap \mathcal{E}^{-}=\emptyset\right)$, strongly connected and structurally balanced; opinions polarize if and only if $\mathcal{E}^{-} \neq \emptyset$, and otherwise consensus is established. If $\mathcal{G}$ is strongly connected but $\mathcal{E}^{+} \cap \mathcal{E}^{-} \neq \emptyset$ or $\mathcal{G}^{ \pm}$is structurally unbalanced, the protocol (4) is stabilizing.

In the case of purely cooperative protocol $\left(a_{j k}(t) \geq 0\right)$, Theorem 3 transforms into the result obtained in [9], [10].

Corollary 2: Cooperative protocol establishes consensus if and only if the graph $G[A(\cdot)]$ is essentially connected.

Our next result addresses the case where $\mathcal{G}$ is not necessarily connected and thus may be decomposed into several disjoint strongly connected components $\mathcal{G}=\mathcal{G}_{1} \cup \mathcal{G}_{2} \cup \ldots \cup \mathcal{G}_{d}, \mathcal{G}_{r}=$ $\left(V_{r}, \mathcal{E}_{r}\right), d \geq 1$. In this case in any component $\mathcal{G}_{r}$, modulus consensus is established, and the type of which depends only on the structure of $\mathcal{G}_{r}$. Let $\mathcal{E}_{r}^{+}:=\mathcal{E}_{r} \cap \mathcal{E}^{+}$and $\mathcal{E}_{r}^{-}:=\mathcal{E}_{r} \cap \mathcal{E}^{-}$. If $\mathcal{E}_{r}^{+} \cap \mathcal{E}_{r}^{-}=\emptyset$, define a signed graph $\mathcal{G}_{r}^{ \pm}$by assigning arcs from $\mathcal{E}_{r}^{+}, \mathcal{E}_{r}^{-}$with weights +1 and -1 respectively.

Theorem 4: For any solution of (5) there exists limits $x_{i}^{\dagger}=\lim _{t \rightarrow \infty} x_{i}(t)$ and $\left|x_{i}^{\dagger}\right|=\left|x_{j}^{\dagger}\right|$ whenever $i$ and $j$ are in the same strongly connected component: $i, j \in V_{r}$. If $\mathcal{E}_{r}^{+} \cap \mathcal{E}_{r}^{-}=\emptyset$ and $\mathcal{G}_{r}^{ \pm}$is structurally balanced, the bipartite consensus is achieved, which comes to consensus if $\mathcal{E}_{r}^{-}=\emptyset$, and otherwise the opinions polarize $\left(V_{r}=V_{r}^{1} \cap V_{r}^{2}\right.$ and $x_{i}^{\dagger}=-x_{j}^{\dagger} \forall i \in V_{r}^{1}, j \in V_{r}^{2}$ ). If $\mathcal{E}_{r}^{+} \cap \mathcal{E}_{r}^{-} \neq \emptyset$ or the graph $\mathcal{G}_{r}^{ \pm}$ is structurally unbalanced, $x_{i}^{\dagger}=0 \forall i \in V_{r}$ that is, dynamics of opinions from $V_{r}$ are stable.

The following criterion of stability is immediate.

Corollary 3: The protocol (4) is stable if and only if for any strongly connected component $\mathcal{G}_{r}$, one has either $\mathcal{E}_{r}^{+} \cap \mathcal{E}_{r}^{-} \neq \emptyset$ or $\mathcal{G}_{r}^{ \pm}$being structurally unbalanced.

Remark 3: Theorems 3 and 4 were proved in [37] in the special case of "type-symmetric" graphs, such that (3) holds for some $K \geq 1$. The main idea of the proof is to show that the latter property remains valid for the "lifted" network, which is purely cooperative and hence can be examined by techniques from [10]. We extend the result from [37] to cut-balanced graphs. Although this extension seems to be provable by techniques from [37], our proof based on [9] is of independent interest; we elaborate mathematical techniques to cope with 
both general and cut-balanced cases in similar ways.

\section{Applications: Nonlinear Protocols}

In this section we apply our results to some types of nonlinear consensus protocols, similar to those from [27], [28].

\section{A. Additive Laplacian protocols}

Our first example concerns nonlinear consensus algorithms that are referred in $[28]$ as the "additive Laplacian feedback schemes". The first of them is

$$
\dot{x}_{i}(t)=\sum_{j=1}^{N}\left|a_{i j}(t)\right|\left(h_{i j}\left(x_{j}(t) \operatorname{sgn} a_{i j}(t)\right)-h_{i j}\left(x_{i}(t)\right)\right) \text {, }
$$

and the second protocol has the form

$$
\left.\dot{x}_{i}(t)=\sum_{j=1}^{N}\left|a_{i j}(t)\right| h_{i j}\left(x_{j}(t) \operatorname{sgn} a_{i j}(t)-x_{i}(t)\right)\right) \forall i .
$$

We adopt the following assumption about the nonlinearities.

Assumption 1: For any $i, j \in 1: N$ the map $h_{i j} \in C^{1}(\mathbb{R})$ is strictly increasing (and hence $h_{i j}^{\prime}>0$ ) with $h_{i j}(0)=0$.

Defining the functions $H_{i j}[y, z]$ as follows: $H_{i j}[y, z]:=$ $\left(h_{i j}(y)-h_{i j}(z)\right) /(y-z)$ for $y \neq z$ and $H_{i j}[z, z]:=h_{i j}^{\prime}(z)$ so that $H_{i j}[y, z]>0$ for any $y, z$. Since $h_{i j} \in C^{1}, H_{i j}$ is easily shown to be a continuous function and $h_{i j}(y)-h_{i j}(z)=$ $H_{i j}[y, z](y-z) \forall y, z$. Under Assumption 1. Theorems 2 and 3 appears to be applicable to the protocols (9), (10) after the standard trick, replacing nonlinearities with the solutiondependent gains, as shown by the following lemma.

Lemma 6: Let $x(t)$ be a solution to system (9), which is defined for $t \geq 0$. Define the matrix $\mathfrak{A}(t)=\left(\mathfrak{a}_{i j}(t)\right)$ by $\mathfrak{a}_{i j}(t):=a_{i j}(t) H_{i j}\left[x_{j}(t) \operatorname{sgn} a_{i j}(t), x_{i}(t)\right]$. Then

$$
\dot{x}(t)=-L[\mathfrak{A}(t)] x(t) .
$$

If the graph $G[A(\cdot)]$ is EQSC, ESC, UQSC, USC, or cutbalanced, then the same is valid for the graph $G[\mathfrak{A}(\cdot)]$. If the matrix $A(\cdot)$ is globally bounded, the same is valid for the matrix $\mathfrak{A}(\cdot)$. These claims also hold for the protocol [10, taking $\mathfrak{a}_{i j}(t):=a_{i j}(t) H_{i j}\left[x_{j}(t) \operatorname{sgn} a_{i j}(t)-x_{i}(t), 0\right]$.

Application of Theorems 2, 3 to (11) yields the following.

Theorem 5: Under Assumption 1, the solutions to systems (9), (10) exist, are unique and infinitely prolongable for any initial condition. If the graph $G[A(\cdot)]$ is USC and $A(\cdot)$ is bounded, or the graph $G[A(\cdot)]$ is ESC and cut-balanced, the protocols (9), 10] establish modulus consensus.

Comparing the result of Theorem 5 with that of [28, Theorem 3,4], one notices that our assumption about the nonlinearities $h_{i j}$ differs from [28], where they are not assumed to be smooth, but only monotonic with some integral constraint. However, unlike [28], functions $h_{i j}$ may be heterogeneous and not necessarily odd; the graph may be time-varying.

Note that Theorem 5 gives only sufficient conditions for modulus consensus. Necessary conditions such as Lemma 5 are not directly applicable since they assume the matrix $A(t)$ to be common for all solutions. Extending the concept of essentially equivalent protocols (see Subsection VI-C) to the nonlinear case, it is possible to get some necessary conditions which are, however, beyond the scope of this paper.

\section{B. Nonlinear Laplacian Flow}

In this subsection we examine the nonlinear consensus protocol similar to that addressed in [28, Section IV-B]:

$$
\dot{x}_{i}(t)=\sum_{j=1}^{N}\left|F_{i j}(t, x)\right|\left(x_{j}(t) \operatorname{sgn} F_{i j}(t, x)-x_{i}(t)\right),
$$

here $i \in 1: N$ and $F_{i j}:[0 ; \infty) \times \mathbb{R}^{N} \rightarrow \mathbb{R}$ are Caratheodory maps, i.e. $F_{i j}(t, \cdot)$ are continuous for a.a. $t$ and $F_{i j}(\cdot, x)$ are measurable for any $x$. We assume also that for any compact set $K \subset[0 ; \infty) \times \mathbb{R}^{N}$ one has

$$
\sup \left\{F_{i j}(t, x): x \in K, t \geq 0\right\}<\infty \quad \forall i, j .
$$

Theorem 6: For any initial condition $x(0)$ a solution of 12 exists for $t \geq 0$ and the matrix $A(t) \triangleq F_{i j}(t, x(t))$ is bounded. If the graph $G[A(\cdot)]$ is USC or ESC and cut-balanced, the protocol (12) establishes modulus consensus.

Although in general it is hard to verify the uniform or essential strong connectivity of $G[A(\cdot)]$, where $A(t)=F_{i j}(t, x(t))$ depends on the concrete solution, in special cases such a property may be proved. For instance, it is implied by the global strong $\varepsilon$-connectivity [28, Section IV-B]: the graph $G\left(\hat{F}_{i j}(t, x)\right)$ is strongly $\varepsilon$-connected for any $t, x$. The result of Theorem 6 extends the result from [28, Section IV-B] in several ways. First of all, it deals with time-variant gains $F_{i j}(t, x)$ and does not require them to have a constant sign. In particular, system (12) does not necessarily generate orderpreserving flow [27]. Moreover, we do not assume that the graph $G[A(t)]$ is weight-balanced which can hardly be provided for nonlinear functions $F_{i j}$. In the case of USC graphs, the balance is not needed at all; in the case of ESC graphs it is replaced by the much weaker cut-balance condition. At last, we relax the connectivity assumption.

\section{PROOFS}

We start with the proof of Lemma 1 and then prove results, concerned with static graphs (Subsection VI-A). To proceed with the case of dynamic graph, we elaborate some useful techniques in Subsections VI-B and VI-C, entailing also Lemmas 3 and 5 . The case of cut-balanced graph is considered in Subsection VI-D. In Subsections VI-E and VI-F we prove the modulus consensus criterion for directed dynamic graphs and its implications, dealing with nonlinear protocols.

Throughout the section, $\Phi\left(t \mid t_{0}\right)$ (where $t, t_{0} \geq 0$ ) stands for the Cauchy evolutionary matrix of the system (4), that is, the solution of the Cauchy problem for (4) with initial data $x\left(t_{0}\right)=x_{0}$ is given by $x(t)=x\left(t \mid t_{0}, x_{0}\right)=\Phi\left(t \mid t_{0}\right) x(0)$.

Proof of Lemma 1. Assume the protocol (4) establishes modulus consensus. Note that since functions $x_{i}(t)$ are continuous, existence of the limits $\lim _{t \rightarrow+\infty}\left|x_{i}(t)\right|=x_{*}$ implies that the limits $\lim _{t \rightarrow+\infty} x_{i}(t)$ also exist (and equal to $\pm x_{*}$ ). Therefore $\Phi(t) \underset{t \rightarrow \infty}{\longrightarrow} \Phi_{*}:=\left[\phi_{1}, \ldots, \phi_{N}\right]$ as $t \rightarrow \infty$, where each column $\phi_{j}$ have entries with equal modules. The same applies for any linear combination $\sum_{j=1}^{N} \alpha_{j} \phi_{j}$. If $\Phi_{*}=0$, the statement of Lemma 1 is evident, taking $v=0$. Assume that 
one of $\phi_{j}$, say, $\phi_{1}$ is nonzero, thus $\phi_{1}=v_{1} \rho$ where $v_{1} \neq 0$ and $\rho$ is a vector with entries \pm 1 . Notice that for any real numbers $\alpha, \beta \neq 0$ we have $|\alpha-\beta| \neq|\alpha+\beta|$. Therefore, if $\phi_{j} \neq 0$ for some $j \neq 1$, all entries of $\phi_{j}-\phi_{1}$ have the same module if and only if $\phi_{j}=v_{j} \rho, v_{j} \neq 0$. If $\phi_{j}=0$, we put by definition $v_{j}=0$. Therefore, $\phi_{j}=v_{j} \rho$ for any $j$ and $\lim _{t \rightarrow \infty} x_{j}(t)=\Phi_{*}=\rho v^{T} x(0)$, where $v:=\left(v_{1}, \ldots, v_{N}\right)^{T}$.

\section{A. Proofs of Lemmas 2,4 and Theorem 1}

Proof of Lemma 2. We use a gauge transformation [28]. Suppose that the graph is structurally balanced; let $V_{1}$ and $V_{2}$ be hostile camps covering all the nodes. Introducing a diagonal matrix $D=\operatorname{diag}\left(d_{1}, \ldots, d_{N}\right)$ by $d_{i}=1$ for $i \in V_{1}$ and $d_{i}=$ -1 for $i \in V_{2}$, one easily shows that the gauge transformation $x \mapsto z:=D x$ transforms the system (4) into

$$
\dot{z}(t)=-L[a b s A] z(t) .
$$

The properties of cooperative protocols 14 are widely known [1], [2], [49]. Since the matrix $B=a b s A$ is non-negative, $L[B]$ has zero eigenvalue with eigenvector $1_{N}$. The algebraic and geometric multiplicities of this eigenvalue coincide [49], and it is simple if and only the graph $G[B]$ is QSC (has oriented spanning tree). If this holds, the protocol (14) establishes consensus and $\exp (-L[B] t) \underset{t \rightarrow+\infty}{\longrightarrow} \overline{1}_{N} v$. Since $L[B]=D L[A] D$ and $G[A]$ is QSC if and only if $G[B]$ is QSC, one immediately obtains the claims of Lemma 2.

Proof of Lemma 4. The proof employs the same idea of the gauge transformation, retracing the arguments from 28 Section III-B-2)]. Suppose the graph $G[A(t)]$ is structurally balanced for any $t$ with static hostile camps $V_{1}$ and $V_{2}$. The gauge transformation $x \mapsto z:=D x$, introduced in the foregoing, transforms the system (4) into (14). Since $G[a b s A(t)]$ is UQSC, the protocol (14) establishes consensus [1], [46] and thus opinions in network (4) polarize.

To proceed with the proof of Theorem 1, note that stability is equivalent to the asymptotic stability of the system (4), which means that $(-L[A])$ is a Hurwitz matrix. By using the Gershgorin disk theorem, it was shown in [28] that all eigenvalues of $L[A]$, except for possibly zero, have positive real parts. Hence protocol is stable if and only if 0 is not an eigenvalue of $L[A]$, i.e. $L[A] \rho=0$ is impossible when $\rho \neq 0$. We need the following simple lemma.

Lemma 7: Let $L[A] \rho=0$ and $|\rho|_{\infty}=1$. Then $V_{1}=\{j:$ $\left.\rho_{j}=1\right\}, V_{2}=\left\{j: \rho_{j}=-1\right\}$ are hostile camps and $V_{*} \triangleq$ $V_{1} \cup V_{2} \neq \emptyset$. If $j \in V_{*}, k \notin V_{*}$, then $a_{j k}=0$.

Proof: By assumption, $\left|x_{k}\right| \leq 1$ for any $k$ and $\left|x_{j}\right|=$ 1 for some $j$, hence $V_{*} \neq \emptyset$. For any such $j$ one has $\rho_{j} \sum_{k \neq j}\left|a_{j k}\right|=\sum_{k \neq j} \rho_{k} a_{j k}$. We note that $\left|\sum_{k \neq j} \rho_{k} a_{j k}\right| \leq$ $\sum_{k \neq j}\left|a_{j k}\right|$. The equality implies, firstly, that $\left|\rho_{k} a_{j k}\right|=\left|a_{j k}\right|$ (i.e. $k \in V_{*}$ or $a_{j k}=0$ ) and secondly, all non-zero terms $\rho_{k} a_{j k}$ have the same sign (coinciding with $\operatorname{sgn} \rho_{j}$ ). In other words, if $j \in V_{*}$, then $a_{j k} \rho_{j} \rho_{k} \geq 0 \forall k \in V_{*}$, and $a_{j k}=0$ when $k \notin V_{*}$, from where the statement is straightforward.

Proof of Theorem 1. Sufficiency in the first statement is immediate from Lemma 77, if no "in-isolated" structurally balanced subgraph exists, then 0 is not an eigenvalue of $(-L[A])$, which therefore is a Hurwitz matrix. Necessity follows from Lemma 4. nodes of the subgraph are independent of the remaining agents and reach bipartite consensus, and hence stability of the whole community is impossible. Lemma 4 implies sufficiency in the second statement. To prove necessity, suppose that bipartite consensus is established. Then 0 is an eigenvalue of $L[A]$ and there exists an eigenvector $\rho$, such that $L[A] \rho=0$, and $|\rho|_{\infty}=1$. Bipartite consensus implies that for this vector one has $\left|\rho_{1}\right|=\ldots=\left|\rho_{N}\right|=1$. Applying Lemma 7 . we obtain the structural balance of $G[A]$ since $V_{*}=1: N$.

\section{B. Ordering permutations}

In this Subsection, we elaborate some useful techniques to be used in the subsequent proofs.

Given a family of scalar functions $f_{1}(t), \ldots, f_{N}(t)$ (where $t \geq 0$ ), let $\left[k^{1}(t), \ldots, k^{N}(t)\right]$ be the ordering permutation, sorting the set $\left\{f_{1}(t), \ldots, f_{N}(t)\right\}$ in the ascending order: $f_{k^{1}(t)}(t) \leq f_{k^{2}(t)}(t) \leq \ldots \leq f_{k^{N}(t)}(t)$. If $f_{j}(t)=f_{k}(t)$ for some $j, k$ and $t$, the permutation is not uniquely defined. The following technical Lemma shows the permutation may always be taken in some regular way.

Lemma 8: Assume that $f_{j}$ are locally Lipschitz. Then there exists such an ordering permutation $k^{1}(t), \ldots, k^{N}(t)$ that $k^{j}(\cdot)$ are measurable, functions $F_{j}(t) \triangleq f_{k^{j}(t)}(t)$ are locally Lipschitz and $\dot{F}_{j}(t)=\dot{f}_{k^{j}(t)}(t)$ for any $j$ and a.a. $t \geq 0$.

To prove Lemma 8 , we need the following proposition.

Lemma 9: Let $f_{*}(t):=\max _{i \in[1: N]} f_{i}(t)$ and $j(t)$ be the index such that $f_{*}(t)=y_{j(t)}(t)$ for a.a. $t$. In the case of nonuniqueness, one always may choose $j(\cdot)$ in a way that it is measurable and $\dot{f}_{*}(t)=\dot{y}_{j(t)}(t)$ for a.a $t$. The claim remains valid, replacing max with min.

Proof: As follows from the generalized version of Danskin theorem [50, Theorem 2.1], $f_{*}(\cdot)$ is locally Lipschitz and $\dot{f}_{*}(t) \in\left\{\dot{y}_{j}(t): y_{j}(t)=f_{*}(t)\right\}$ for a.a. $t \geq 0$. The Filippov-Castaing measurable selector theorem (see e.g. [51 Theorem 1]) yields that a measurable function $j(t)$ exists such that $\dot{f}_{*}(t)=\dot{y}_{j(t)}(t)$ and $y_{j(t)}(t)=f_{*}(t)$. The last claim is proved by replacement $f_{j} \mapsto-f_{j}$.

Proof of Lemma 8. The proof is via induction on $N$. For $N=1$, the claim is evident. Let it be true for some $N$, and let $f_{1}(\cdot), \ldots, f_{N+1}(\cdot)$ be locally Lipschitz. The ordering of the first $N$ functions $z_{1}(\cdot), \ldots, z_{N}(\cdot)$ are locally Lipschitz by the induction hypothesis. The recursion $y_{N+1}^{0}(t):=y_{N+1}(t)$,

$$
\begin{gathered}
y_{N+1}^{\nu}(t):=\max \left\{z_{\nu}(t) ; y_{N+1}^{\nu-1}(t)\right\}, \\
\widehat{z}_{\nu}(t):=\min \left\{z_{\nu}(t) ; y_{N+1}^{\nu-1}(t)\right\}, \nu \in 1: N
\end{gathered}
$$

results in the ordering $\widehat{z}_{1}(t) \leq \ldots \leq \widehat{z}_{N}(t) \leq \widehat{z}_{N+1}(t):=$ $y_{N+1, N}(t)$ of the entire set $y_{1}(\cdot), \ldots, y_{N+1}(\cdot)$. By applying Lemma 9 at every recursion step, we see that $\widehat{z}_{\nu}(\cdot)$ are locally Lipschitz. For any $\nu=1, \ldots, N$, the sequence

$z_{\nu}(t):=\left[\widehat{z}_{1}(t), \widehat{z}_{2}(t), \ldots, \widehat{z}_{\nu}(t), z_{\nu+1}(t), \ldots, z_{N}(t), y_{N+1}^{\nu}(t)\right]$

is obtained from $z_{\nu-1}$ via a permutation $J_{\nu}(t)$ of indices, which either is the identity one or exchanges the places of the $\nu$ th and $(N+1)$ th entries and may be chosen measurable. By Lemma 9 , the sequence $z_{\nu}^{\prime}(t)$ that results from replacement of 
any function in 15 by its derivative is related to $z_{\nu-1}^{\prime}(t)$ by the same permutation for a.a. $t$. The sequence $z_{0}(t)$ is obtained from $y(t):=\left[y_{1}(t), \ldots, y_{N+1}(t)\right]$ via a permutation of indices $\mathcal{K}(t)=\left[k^{1}(t), \ldots, k^{N}(t), N+1\right]$. By the induction hypothesis, $\mathcal{K}(t)$ also relates $z_{0}^{\prime}(t)$ and $y^{\prime}(t):=\left[y_{1}^{\prime}(t), \ldots, y_{N+1}^{\prime}(t)\right]$ for a.a. $t$. Then $\mathcal{K}_{N+1}(t)=J_{N} \circ \cdots \circ J_{1} \circ \mathcal{K}(t)$ transforms $y^{\prime}(t)$ into $Z_{N}^{\prime}(t)$ for a.a. $t$, which proves the induction step.

\section{Some Technical Lemmas and Proofs of Lemmas 35}

To start with, we note that since $a_{j k}(t)$ are locally bounded, this also holds for $\dot{x}_{j}$ and therefore $x_{j}(\cdot)$ are locally Lipschitz. For any solution $x(t)$ of 4 we introduce functions

$$
\chi_{j}(t):=\left|x_{j}(t)\right|, \theta_{i j}(t):=\operatorname{sgna}_{i j}(t) \operatorname{sgn} x_{i}(t) \operatorname{sgn} x_{j}(t) .
$$

The following lemma gives a useful interpretation of dynamics (4) in terms of the moduli functions $\chi_{k}$.

Lemma 10: The functions $\chi_{j}$ are locally Lipschitz, thus absolutely continuous. For a.a. $t \geq 0$ and any $k$ one has

$$
\dot{\chi}_{k}(t)=\sum_{i=1}^{N}\left|a_{k i}(t)\right|\left[\chi_{i}(t) \theta_{k i}(t)-\chi_{k}(t)\right] .
$$

(since $\chi_{k} \geq 0$, it has sign $\operatorname{sgn} \chi_{k}(t)$ equal to 0 or 1 ).

Proof: Since $a_{j k}$ are assumed to be locally bounded, $x_{k}(\cdot)$ are locally Lipschitz. The same applies to $\chi_{k}(\cdot)$ since $\left|\chi_{k}\left(t_{1}\right)-\chi_{k}\left(t_{2}\right)\right|=|| x_{k}\left(t_{1}\right)|-| x_{k}\left(t_{2}\right)|| \leq\left|x_{k}\left(t_{1}\right)-x_{k}\left(t_{2}\right)\right|$. Therefore $\chi_{k}$ are absolutely continuous and for a.a. $t>0$ the derivative $\dot{\chi}_{k}(t)$ exists. For such $t$ we immediately have $\chi_{k}(t)=0 \Longrightarrow \dot{\chi}_{k}(t)=0$ by the Fermat theorem since 0 is the global minimum of $\chi_{k}$, which proves (16) (indeed, $x_{k}(t)=0$ implies that $\theta_{k i}(t)=0$ for any $i$ ). Let $\chi_{k}(t)>0$. Since $\operatorname{sgn} x_{k}(s)=\operatorname{sgn} x_{k}(t)$ for $s \approx t$, one has

$$
\begin{aligned}
\dot{\chi}_{k}(t) & =\dot{x}_{k}(t) \operatorname{sgn}_{k}(t) \stackrel{\underline{5}}{ } \\
& \underline{\underline{5}} \sum_{i=1}^{N}\left|a_{k i}(t)\right|\left[x_{i}(t) \operatorname{sgn} a_{k i}(t) \operatorname{sgn} x_{k}(t)-\chi_{k}(t)\right],
\end{aligned}
$$

which proves (16) since $x_{i}(t)=\chi_{i}(t) \operatorname{sgn} x_{i}(t)$.

Henceforth, we fix some ordering permutation $k^{1}(t), \ldots, k^{N}(t)$ for the family $\chi_{1}(t), \ldots, \chi_{N}(t)$ (see Subsect VI-B and put $M_{j}(t)=\chi_{k^{j}(t)}$, in particular, $M_{N}(t)=\max _{j}\left|x_{j}(t)\right|$. Combining Lemmas 10 and 8 , one gets the following.

Lemma 11: The function $M_{N}(t)=\max _{i \in 1: N}\left|x_{i}(t)\right|$ is nonincreasing and hence $\int_{0}^{\infty}\left|\dot{M}_{N}(t)\right| d t<\infty$. For $\tilde{a}_{j i}:=a_{k^{j}} k^{i}$, $\tilde{\theta}_{j i}:=\theta_{k^{j} k^{i}}$, for a.a. $t \geq 0$ and any $j \in 1: N$ one has

$$
\dot{M}_{j}(t)=\sum_{i=1}^{N}\left|\tilde{a}_{j i}(t)\right|\left[M_{i}(t) \tilde{\theta}_{j i}(t)-M_{j}(t)\right] .
$$

Proof: Combining (16) with Lemma 8, we get

$$
\begin{aligned}
\dot{M}_{j}(t) & =\sum_{i=1}^{N}\left|a_{k^{j}}(t)\right|\left[\chi_{i}(t) \theta_{k^{j} i}(t)-M_{j}(t)\right] \\
& =\sum_{i=1}^{N}\left|a_{k^{j} k^{i}}(t)\right|\left[\chi_{k^{i}}(t) \theta_{k^{j} k^{i}}(t)-M_{j}(t)\right],
\end{aligned}
$$

from where (17) follows since $M_{i}=\chi_{k^{i}}$. Using (17), one has $\dot{M}_{N}(t) \leq 0$ for a.a. $t \geq 0$ since $\left|\tilde{\theta}_{i j}\right| \leq 1$ and $M_{j} \leq$ $M_{N}$ for any $j$ thus $M_{N}(\cdot)$ is non-increasing. We have also $\int_{0}^{\infty}\left|\dot{M}_{N}(t)\right| d t=M(0)-\inf _{t \geq 0} M(t) \leq M(0)$.

We also require one additional simple tool which allows to examine the behavior of system (5) by comparing it with a simpler system, obtained by ignoring all inessential interactions between the agents. Consider a protocol

$$
\dot{\xi}(t)=-L[\mathfrak{A}(t)] \xi(t)
$$

where $\mathfrak{A}(t)=\left(\mathfrak{a}_{i j}(t)\right)$ is locally bounded. We say the protocol (18) is essentially equivalent to (4) if

$$
\int_{0}^{\infty}\left|\mathfrak{a}_{i j}(t)-a_{i j}(t)\right| d t<\infty \quad \forall i, j .
$$

We are going to show that the essentially equivalent protocol provides the same limit sets for the solutions. Let $B_{1}:=\{x \in$ $\left.\mathbb{R}^{N}:|x|_{1} \leq 1 \forall i\right\}$ be a unit ball in the $|\cdot|_{\infty}$-norm, positively invariant by Lemma 11: $x\left(t_{0}\right) \in B_{1} \Longrightarrow x(t) \in B_{1} \forall t \geq t_{0}$.

Definition 3: Let $\Omega_{t_{0}, x_{0}}:=\left\{y \in \mathbb{R}^{N}: \exists t_{n} \rightarrow \infty\right.$ : $\left.\left.x\left(t_{n} \mid t_{0}, x_{0}\right) \underset{n \rightarrow \infty}{\longrightarrow} y\right)\right\}$. We call the set $\Omega:=\overline{\bigcup_{t_{0}, x_{0}} \Omega_{t_{0}, x_{0}}} \subseteq B_{1}$ the $\Omega$-set of the system (4) (the union is over $t_{0} \geq 0, x_{0} \in B_{1}$ ).

Lemma 12: Suppose the protocols (4) and (18) are essentially equivalent. Then for any $\varepsilon>0$ there exists $T_{0}=T_{0}(\varepsilon)$ such that $\xi\left(T_{0}\right)=x\left(T_{0}\right) \in B \Longrightarrow|x(t)-\xi(t)|_{\infty} \leq \varepsilon \forall t \geq T_{0}$. In particular, the systems (4) and (18) have equal $\Omega$-sets.

Proof: Since $x\left(t_{0}\right) \in B_{1} \Longrightarrow x(t) \in B_{1} \forall t \geq t_{0}$ thanks to Lemma 11, one has $\left|\Phi\left(t \mid t_{0}\right)\right|_{\infty} \leq 1 \forall t_{0} \geq 0 \forall t \geq t_{0}$. Let $T_{0}>$ 0 be so large that $\int_{T_{0}}^{\infty}|L[A(t)]-L[\mathfrak{A}(t)]|_{\infty} d t<\varepsilon$. Applying Lemma 11 to (18) implies that $\xi\left(T_{0}\right) \in B \Longrightarrow \xi(t) \in B \forall t \geq$ $T_{0}$, and hence $\Delta(t):=(L[A(t)]-L[\mathfrak{A}(t)]) \xi(t)$ satisfies the inequality $\int_{T_{0}}^{\infty}|\Delta(t)|_{\infty} d t<\varepsilon$. Since $\xi^{\prime}(t)=-L[A(t)] \xi+$ $\Delta(t)$, condition $x\left(T_{0}\right)=\xi\left(T_{0}\right) \in B$ implies

$$
\xi(t)-x(t)=\xi(t)-\Phi\left(t \mid T_{0}\right) \xi\left(T_{0}\right)=\int_{T_{0}}^{t} \Phi(t \mid s) \Delta(s) d s
$$

and therefore $|\xi(t)-x(t)|_{\infty} \leq \int_{T_{0}}^{t}|\Phi(t \mid s)|_{\infty}|\Delta(s)|_{\infty} d s<\varepsilon$. This proves the first claim from where the second one immediate follows: indeed, for any solution $x\left(t \mid t_{0}, x_{0}\right)$ with $t_{0} \geq 0, x_{0} \in B$ and any $\varepsilon>0$ one can find $T_{0}$ such that $\left|\xi\left(t \mid T_{0}, \xi_{0}\right)-x\left(t \mid t_{0}, x_{0}\right)\right|_{\infty}=\left|\xi\left(t \mid T_{0}, \xi_{0}\right)-x\left(t \mid T_{0}, \xi_{0}\right)\right|_{\infty} \leq \varepsilon$ for any $t \geq T_{0}$, where $\xi_{0}:=x\left(T_{0} \mid t_{0}, x_{0}\right) \in B$. Therefore, any set $\Omega_{t_{0}, x_{0}}$ from Definition 3 belongs to the $\Omega$-set of (18) and thus the whole $\Omega$-set of (4) belongs to the $\Omega$-set of $(18)$. The inverse inclusion is proved in the same way.

Remark 4: Such properties of the protocol as modulus consensus, stability, bipartite consensus, and "partial" modulus consensus (modulus agreement among a subgroup of agents) in fact depend only on the $\Omega$-set. For instance, modulus consensus is established if and only if the $\Omega$-set is comprised of the set $\left\{x \in B:\left|x_{1}\right|=\ldots=\left|x_{N}\right|\right\}$. Lemma 12 implies that those properties are preserved, replacing the protocol with essentially equivalent one.

We are now going to prove Lemmas 3 , 5 Lemma 3 immediately follows from Lemma 11 since $M_{N}(t)=\max _{j}\left|x_{j}(t)\right|$.

Proof of Lemma 5. Suppose the protocol (4) establishes bipartite consensus. Lemma 12 and Remark 4 show that, 
without loss of generality, one may assume $a_{j k} \equiv 0$ unless $j$ essentially interacts with $k$, i.e. $(j, k) \in \mathcal{E}[A(\cdot)]$. If the topology is not EQSC, then the graph $\mathcal{G}[A(\cdot)]$ is not QSC and thus, as shown in [5], there exist non-empty disjoint subsets $V_{1}, V_{2} \subset 1: N$ that has no incoming arcs: $a_{j k}=0$ if $j \in V_{1}, k \notin V_{1}$ or $j \in V_{2}, k \notin V_{2}$. Therefore, the opinions of agents from $V_{1}$ are independent on the opinions of agents from $V_{2}$, and hence bipartite consensus is impossible.

\section{Proof of Theorems 3,4 and Corollary 3}

Henceforth $G[A(\cdot)]$ is cut-balanced. The cornerstone of the proofs is the following lemma, based on Lemma 11

Lemma 13: For a given solution of (4), let $\tilde{\eta}_{j i}(t):=$ $\left|\tilde{a}_{j i}(t)\right|\left(\tilde{\theta}_{j i}(t) M_{i}(t)-M_{j}(t)\right)$, where $\tilde{\theta}_{j i}$ are the same as in (16). Then $\tilde{\eta}_{j i} \in L^{1}[0 ; \infty]$ for any $i, j$, so that $\dot{M}_{j} \in L^{1}[0 ; \infty]$.

Proof: Lemma 11, which is valid for any dynamic graph, shows that $\tilde{\eta}_{N 1}, \ldots, \tilde{\eta}_{N, N-1} \in L^{1}[0 ; \infty]$ since $\tilde{\eta}_{N j} \leq 0$ and $\dot{M}_{N} \in L^{1}$. We are going to show that $\tilde{\eta}_{N-1, j} \in L^{1}$.

Note that $\left|\tilde{\eta}_{N j}(t)\right| \geq\left|\tilde{a}_{N j}(t)\right|\left(M_{N}(t)-M_{N-1}(t)\right)$ for any $j<N$. Indeed, $\left|\tilde{\theta}_{N, j} M_{j}-M_{N}\right| \geq\left|M_{N}\right|-\left|\tilde{\theta}_{N, j} M_{j}\right| \geq$ $M_{N}-M_{N-1}$ since $M_{j} \leq M_{N-1}$. Applying the definition of cut-balance (2) to $V^{\prime}=\left\{k^{1}, k^{2}, \ldots, k^{N-1}\right\}$ and $V^{\prime \prime}=$ $\left\{k^{N}\right\}$, one obtains $\sum_{j<N}\left|\tilde{a}_{j N}(t)\right| \leq K \sum_{j<N}\left|\tilde{a}_{N j}(t)\right|$. Therefore the function $S_{N}(t) \triangleq\left(M_{N}(t)-M_{N-1}(t)\right) \sum_{j=1}^{N-1}\left|\tilde{a}_{j N}(t)\right|$ is summable. Note that if $\tilde{\eta}_{N-1, N}(t)>0$ then $\tilde{\theta}_{N-1, N}(t)=1$ and $\tilde{\eta}_{N-1, N}(t)=\left|a_{N-1, N}(t)\right|\left(M_{N}(t)-M_{N-1}(t)\right)$. Thus, $\tilde{\eta}_{N-1, N}^{+}(t) \leq S_{N}(t)$ and hence $\tilde{\eta}_{N-1, N}^{+} \in L^{1}$. By invoking (17) for $j=N-1$, one obtains that

$$
\dot{M}_{N-1}(t)=-\tilde{\eta}_{N-1, N}^{-}(t)+\sum_{j=1}^{N-2} \tilde{\eta}_{N-1, j}(t)+\tilde{\eta}_{N-1, N}^{+}(t) .
$$

Since $-\tilde{\eta}_{N-1, N}^{-}(t) \leq 0$ and $\tilde{\eta}_{N-1, j}(t) \leq 0$ for any $t \geq 0$ and the last term in 190 is $L^{1}$-summable, we either have $\tilde{\eta}_{N-1, N}^{-}, \tilde{\eta}_{N-1, j} \in L^{1}$ or $\int_{0}^{\infty} \dot{M}_{N-1}(t) d t=-\infty$. The latter is impossible since $M_{N-1}(t) \geq 0$. Thus $\tilde{\eta}_{N-1, j} \in L^{1}[0 ; \infty] \forall j$.

Our next step is to prove that $\tilde{\eta}_{N-2, j} \in L^{1}$ for any $j$. We note that for any $j \leq N-2$ and $r=N-1, N$ we have $\left|\tilde{\eta}_{r j}(t)\right| \geq\left|\tilde{a}_{r j}(t)\right|\left(M_{N-1}(t)-M_{N-2}(t)\right)$. Applying (2) to $V^{\prime}=\left\{k^{1}, k^{2}, \ldots, k^{N-2}\right\}$ and $V^{\prime \prime}=\left\{k^{N-1}, k^{N}\right\}$, we obtain that $\sum_{j=1}^{N-2} \sum_{r=N-1}^{N}\left|\tilde{a}_{j r}(t)\right| \leq$ $K \sum_{j=1}^{N-2} \sum_{r=N-1}^{N}\left|\tilde{a}_{r j}(t)\right|$ and hence the function $S_{N-1}(t) \triangleq\left(M_{N-1}(t)-M_{N-2}(t)\right) \sum_{j=1}^{N-2} \sum_{r=N-1}^{N}\left|\tilde{a}_{j r}(t)\right|$ belongs to $L^{1}$. If $\tilde{\eta}_{N-2, N-1}(t)>0$, one has $\tilde{\eta}_{N-2, N-1}(t)=\left|\tilde{a}_{N-2, N-1}(t)\right|\left(M_{N-1}-M_{N-2}(t)\right)$. Therefore, $\tilde{\eta}_{N-2, N-1}^{+} \leq S_{N-1}$. Analogously, if $\tilde{\eta}_{N-2, N}(t)>0$ then $\tilde{\eta}_{N-2, N}(t)=\left|\tilde{a}_{N-2, N}(t)\right|\left(M_{N}(t)-M_{N-1}(t)+\right.$ $\left.M_{N-1}-M_{N-2}(t)\right)$ and hence $\tilde{\eta}_{N-2, N}^{+} \leq S_{N-1}+S_{N}$. Thus
$\tilde{\eta}_{N-2, N}^{+}, \tilde{\eta}_{N-2, N-1}^{+} \in L^{1}[0 ; \infty]$. Applying (17), we get

$$
\begin{array}{r}
\dot{M}_{N-2}(t)=-\tilde{\eta}_{N-2, N-1}^{-}(t)-\tilde{\eta}_{N-2, N}^{-}(t)+\sum_{j=1}^{N-3} \tilde{\eta}_{N-2, j}(t)+ \\
+\tilde{\eta}_{N-2, N-1}^{+}(t)+\tilde{\eta}_{N-2, N}^{+}(t) .
\end{array}
$$

Since $-\tilde{\eta}_{N-2, N}^{-}(t) \leq 0,-\tilde{\eta}_{N-2, N-1}^{-}(t) \leq 0$ and $\tilde{\eta}_{N-2, j}(t) \leq$ 0 for $j<N-2$, then either all of these functions are summable or $\int_{0}^{\infty} \dot{M}_{N-2}(t) d t=-\infty$. The latter is impossible since $M_{N-2}(t) \geq 0$. Therefore, $\tilde{\eta}_{N-2, j} \in L^{1}[0 ; \infty] \forall j$.

Applying the same procedure, one proves that $S_{N-2}(t) \triangleq$ $\left(M_{N-2}(t)-M_{N-3}(t)\right) \sum_{j=1}^{N-2} \sum_{r=N-2}^{N}\left|\tilde{a}_{j r}(t)\right|$ is summable and $\tilde{\eta}_{N-3, j} \in L^{1}[0 ; \infty] \forall j$, and so on, $\tilde{\eta}_{i j} \in L^{1}[0 ; \infty]$.

Corollary 4: For any solution of (4) one has $\eta_{i j}:=$ $\left|a_{i j}\right|\left(\theta_{i j} \chi_{j}-\chi_{i}\right) \in L^{1}[0 ; \infty]$ and $\dot{\chi}_{i} \in L^{1}[0 ; \infty]$, so the finite limits $\chi_{i}^{0}=\lim _{t \rightarrow+\infty} \chi_{i}(t)$ and $x_{i}^{0}:=\lim _{t \rightarrow+\infty} x_{i}(t)= \pm \chi_{i}^{0}$ exist. If $(i, j) \in \mathcal{E}[A(\cdot)]$ then $\left|x_{i}^{0}\right|=\left|x_{j}^{0}\right| ;$ moreover, $x_{i}^{0}=x_{j}^{0}$ when $(i, j) \in \mathcal{E}^{+}[A(\cdot)]$ and $x_{i}^{0}=-x_{j}^{0}$ when $(i, j) \in \mathcal{E}^{-}[A(\cdot)]$; as a consequence, $x_{i}^{0}=x_{j}^{0}=0$ if $(i, j) \in \mathcal{E}^{+}[A(\cdot)] \cap \mathcal{E}^{-}[A(\cdot)]$.

Proof: Since $k^{1}, \ldots, k^{N}$ is just a permutation of the set $1: N$, we have $\sum_{i, j}\left|\eta_{i j}\right|=\sum_{i, j}\left|\tilde{\eta}_{i j}\right|$, and hence $\eta_{j i} \in L^{1}[0 ; \infty]$ for any $i, j$. From $(16)$ one immediately obtains that $\dot{\chi}_{i} \in L^{1}[0 ; \infty]$ for any $i$, from where the existence of the finite limits $\chi_{i}^{0}:=\lim _{t \rightarrow+\infty} \chi_{i}(t)$ is immediate. The limits $x_{i}^{0}=\lim _{t \rightarrow+\infty} x_{i}(t)=\stackrel{t \rightarrow+\infty}{ \pm} \chi_{i}^{0}$ exist since $x_{i}$ are continuous. From $\left|\eta_{i j}(t)\right| \geq\left|a_{i j}(t)\right|\left|\chi_{j}(t)-\chi_{i}(t)\right|$ we know that if $\delta:=\left|\chi_{i}^{0}-\chi_{j}^{0}\right|>0$, for large $t>0$ one has $\left|\eta_{i j}(t)\right| \geq\left|a_{i j}(t)\right| \delta / 2$ and thus $\int_{0}^{\infty}\left|a_{i j}(t)\right| d t<\infty\left(a_{i j}\right.$ are locally bounded). Therefore, $\chi_{i}^{0}=\chi_{j}^{0}$ whenever $i$ and $j$ essentially interact. Suppose that $x_{i}^{0}=x_{j}^{0} \neq 0$. As $t \rightarrow \infty$, one has $\operatorname{sgn} x_{i}(t)=\operatorname{sgn} x_{i}^{0}=\operatorname{sgn} x_{j}(t)$ and thus if $a_{j k}(t)<0$, we have $\eta_{i j}(t)=\left|a_{i j}(t)\right|\left(-x_{i}(t)-x_{j}(t)\right)$. Thus $\left|\eta_{i j}(t)\right| \geq\left|a_{i j}^{-}(t)\right|\left|x_{i}^{0}\right|$ for $t>0$ sufficiently large, from where one has that $\int_{i j}\left|a_{i j}^{-}(t)\right| d t<\infty$. So if the agents essentially compete, the option $x_{i}^{0}=x_{j}^{0} \neq 0$ is impossible, and thus $x_{i}^{0}=-x_{j}^{0}$ (with possibility of $x_{i}^{0}=x_{j}^{0}=0$ ). Analogously, one can easily show that $\left|\eta_{i j}(t)\right| \geq\left|a_{i j}^{+}(t)\right|\left|x_{i}^{0}\right|$ for $t>0$ sufficiently large if $x_{i}^{0}=-x_{j}^{0} \neq 0$ which proves the essential cooperation excludes the possibility of $x_{i}^{0}=-x_{j}^{0} \neq 0$ and thus $x_{i}^{0}=x_{j}^{0}$. At last, simultaneous essential cooperation and essential competition imply that $x_{i}^{0}=x_{j}^{0}=-x_{j}^{0}=0$.

Corollary 5: If the network topology is ESC, the protocol (4) establishes modulus consensus $\left(\chi_{1}^{0}=\ldots=\chi_{N}^{0}\right)$, and $\chi_{j}^{0}=0$ unless $\mathcal{G}^{ \pm}$is well-defined $\left(\mathcal{E}^{+} \cap \mathcal{E}^{-}=\emptyset\right)$ and SB.

Proof: The first statement immediately follows from Corollary 4 since for any path $i_{1}, i_{2}, \ldots, i_{r}$ in $\mathcal{G}$ one has $\chi_{i_{1}}^{0}=\ldots=\chi_{i_{r}}^{0}$. Assume that for some initial vector $x(0)$ one has $\chi_{i}^{0} \neq 0$. Corollary 4 implies that no pair of agents may be both essentially cooperative and essentially competitive, and thus $\mathcal{E}^{+} \cap \mathcal{E}^{-}=\emptyset$ so the signed graph $\mathcal{G}^{ \pm}$is well-defined. We have to show $\mathcal{G}^{ \pm}$is structurally balanced, in other words [17], [28], has no negative cycles. Indeed, the weight of any arc $(i, j) \in \mathcal{E}$ is $s_{i j}=1$ if $(i, j) \in \mathcal{E}^{+}$(hence $x_{i}^{0}=x_{j}^{0}$ ) and $s_{i j}=$ -1 if $(i, j) \in \mathcal{E}^{-}$(and thus $\left.x_{i}^{0}=-x_{j}^{0}\right)$ so that $x_{i}^{0} x_{j}^{0} s_{i j} \geq 0$. Given a cycle $i_{1}, \ldots, i_{n}=i_{1}$, Multiplying the inequalities 
$x_{i_{k}}^{0} x_{i_{k+1}}^{0} s_{i_{k} i_{k+1}} \geq 0$, where $i_{1}, i_{2}, \ldots, i_{n}=i_{1}$ is a cycle and $k \in 1:(n-1)$, one has $s_{i_{1} i_{2}} s_{i_{2} i_{3}} \ldots s_{i_{n-1} i_{n}}\left(x_{1}^{0} \ldots x_{n}^{0}\right)^{2} \geq 0$ which means that the cycle is positive.

Proof of Theorem 3. The necessity in the first statement part follows from Lemma 5 and Corollary 5, indeed, the ESC condition is necessary for bipartite consensus independent of the cut-balance property. Under the ESC condition, bipartite consensus is possible only when $\mathcal{G}^{ \pm}$exists and is SB. To prove sufficiency, suppose that $\mathcal{G}^{ \pm}$is well-defined, strongly connected and structurally balanced. Thanks to Corollary 5 . the protocol (4) establishes modulus consensus, and it remains to show it is bipartite consensus. Indeed, consider the protocol (18), where $\mathfrak{A}(t)=\left(\mathfrak{a}_{j k}(t)\right)$ and $\mathfrak{a}_{j k}(t)=a_{j k}^{+}(t)$ when $(j, k) \in \mathcal{E}^{+}, \mathfrak{a}_{j k}(t)=a_{j k}^{-}(t)$ when $(j, k) \in \mathcal{E}^{-}$, and otherwise $\mathfrak{a}_{j k} \equiv 0$. The protocol (18) is equivalent to the protocol (4) and hence establishes modulus consensus of the same type as (18) by Lemma 12 and Remark 4 Let $V_{1}, V_{2}$ be the hostile camps of the graph $\mathcal{G}^{ \pm}$, covering all its nodes. Taking $x_{j}=+1$ for $j \in V_{1}$ and $x_{j}=-1$ for $j \in V_{2}$, one can show that $x=\left(x_{1}, \ldots, x_{N}\right)^{T}$ is an equilibrium point for $(18)$ and hence bipartite consensus is reached. Accordingly to Corollary 4. $x_{i}^{0}=x_{j}^{0}$ if $i, j \in V_{r}^{1}$ or $i, j \in V_{r}^{2}$, and $x_{i}^{0}=-x_{j}^{0}$ whenever $i \in V_{r}^{1}, j \in V_{r}^{2}$; therefore, opinions polarize unless $E_{r}^{-}=\emptyset$. The claim about stability also follows from Corollary 4.

Remark 5: It may seem that Theorem 3 may be proved by applying the result for unsigned graphs [10] to the system, obtained from the protocol $[18$ just constructed via the gauge transformation (as Lemma 4 was derived from the relevant result on cooperative agents). Unfortunately, this is not the case. The problem is that the graph $G[\mathfrak{A}(\cdot)]$ is no longer cutbalanced. This property depends not only on the integrals $\int_{0}^{\infty}\left|a_{j k}(s)\right| d s$, but on the whole function $A(t)$, and is lost after removing inessential interactions.

Proof of Theorem 4. The first and the last claims follow from Corollary 4 . The second claim is proved by passing to an auxiliary protocol $(18)$, constructed in the proof of Theorem 3 . Let $V_{r}^{1}$ and $V_{r}^{2}$ be hostile camps in $\mathcal{G}_{r}^{ \pm}$, then taking $x_{i}=+1$ for $i \in V_{r}^{1}, x_{i}=-1$ for $i \in V_{r}^{2}$ and $x_{i}=0$; otherwise, we get an equilibrium point of (18), and therefore, modulus consensus in the subcommunity $V_{r}$ is bipartite. Thanks to Corollary 4 $x_{i}^{0}=x_{j}^{0}$ if $i, j \in V_{r}^{1}$ or $i, j \in V_{r}^{2}$, and $x_{i}^{0}=-x_{j}^{0}$ whenever $i \in V_{r}^{1}, j \in V_{r}^{2}$; therefore, opinions polarize unless $E_{r}^{-}=\emptyset$, when bipartite consensus is established.

\section{E. Proof of Theorem 2 and Corollary 1}

We start with some useful estimates for the solutions.

Lemma 14: Suppose that $A:\left[t_{0} ; t_{1}\right] \rightarrow \mathbb{R}^{N}$ is a matrixvalued function, $|A(t)|_{\infty} \leq R$ for a.a. $\in\left[t_{0} ; t_{1}\right]$ and $\theta_{0} \triangleq$ $e^{-R\left(t_{1}-t_{0}\right)}$. For any solution of (4) one has

$\left|x_{k}(t)\right| \leq \theta_{0}\left|x_{k}\left(t_{0}\right)\right|+\left(1-\theta_{0}\right)\left|x\left(t_{0}\right)\right|_{\infty} \forall k \in 1: N \forall t \in\left[t_{0} ; t_{1}\right]$

Proof: Let $M \triangleq\left|x\left(t_{0}\right)\right|_{\infty}$. Thanks to Lemma 3 , one has $\left|x_{j}(t)\right| \leq M$ for any $j$, $t$. Let $s_{k}(t) \triangleq \sum_{j=1}^{N}\left|a_{k j}(t)\right|$ and $S_{k}(t) \triangleq \int_{t_{0}}^{t} s_{k}(\xi) d \xi$. By assumption, $s_{k}(t) \leq R$ and hence
$S_{k}(t) \leq R\left(t_{1}-t_{0}\right) \forall t \in\left[t_{0} ; t_{1}\right]$. From (16) one derives that

$$
\chi_{k}(t) \leq\left[\chi_{k}\left(t_{0}\right)+\int_{0}^{t} e^{S_{k}(\tau)} \sum_{i=1}^{N}\left|a_{k i}(\tau)\right| \chi_{i}(\tau) d \tau\right] e^{-S_{k}(t)} .
$$

Since $\chi_{i}(\tau) \leq M$, one has $\sum\left|a_{k i}(\tau)\right| \chi_{i}(\tau) \leq M s_{k}(\tau)$ and $\chi_{k}(t) \leq M+\left[\chi_{k}\left(t_{0}\right)-M\right] e^{-S_{k}(t)} \leq M+\left[\chi_{k}\left(t_{0}\right)-M\right] \theta_{0}$.

Lemma 15: Under assumptions of Lemma 14, suppose the graph $G\left[\int_{t_{0}}^{t_{1}} a b s A(t) d t\right]$ is strongly $\varepsilon$-connected and $\theta \triangleq$ $\varepsilon \theta_{0}^{2}<1$. Let $V \subsetneq 1: N$ be a non-empty set, $\left|x\left(t_{0}\right)\right|_{\infty}=M$ and $\max _{j \notin V}\left|x_{j}\left(t_{0}\right)\right|=M^{\prime}$. Then there exists $k \in V$ such that

$$
\left|x_{k}\left(t_{1}\right)\right| \leq \theta M^{\prime}+(1-\theta) M
$$

Proof: Since the graph $G\left[\int_{t_{0}}^{t_{1}} a b s A(t) d t\right]$ is strongly $\varepsilon$-connected, there exist $i \notin V$ and $k \in V$ such that $\int_{t_{0}}^{t_{1}}\left|a_{k i}(t)\right| d t \geq \varepsilon$. From 20 one has $\chi_{j}(t) \leq M+\left(M^{\prime}-\right.$ $M) \theta \forall j \notin V$ and $\chi_{j}(t) \leq M \forall j \in V$, which entails that $e^{S_{k}(\tau)} \sum_{i=1}^{N}\left|a_{k i}(\tau)\right| \chi_{i}(\tau) \leq M e^{S_{k}(\tau)} s_{k}(\tau)-\left(M-M^{\prime}\right) \theta \varepsilon$ (we used that $e^{S_{k}(\tau)} \geq 1$ ). Inequality (21) yields now that

$$
\chi_{k}\left(t_{1}\right) \leq M+\left(M^{\prime}-M\right) \theta_{0} \varepsilon e^{-S_{k}\left(t_{1}\right)} \leq M+\left(M^{\prime}-M\right) \theta,
$$

since $e^{S_{k}\left(t_{1}\right)} \leq e^{R\left(t_{1}-t_{0}\right)}=\theta_{0}$.

Proof of Theorem 2. By assumption, there exist $R, \varepsilon, T>0$ such that $|A(t)|_{\infty} \leq R$ and the graph $G_{t}=$ $G\left[\int_{t}^{t+T} a b s A(\tau) d \tau\right]$ is strongly $\varepsilon$-connected for any $t \geq 0$. Let $\theta_{0}=e^{-R T}$ and $\theta=\varepsilon \theta_{0}^{2}$, without loss of generality we may assume that $\theta<1$.

We know from Lemma 3 that the maximal modulus $M_{N}(t)$ always has a limit: $M_{N}(t) \rightarrow M_{*}$ as $t \rightarrow+\infty$ (here we use the notation introduced in Subsection VI-C so that $M_{j}(t)$ is the $j$-th modulus in the ascending order, $M_{j}(t)=\left|x_{k^{j}(t)}(t)\right|$ ). Our goal is to show that $M_{j}(t) \rightarrow M_{*}$ via induction by $j=$ $N, N-1, \ldots, 1$. If $M_{*}=0$ the latter claim is trivial since $0 \leq M_{j}(t) \leq M_{N}(t)$; hence we may assume that $M_{*}>0$.

For $j=N$ our claim holds by definition of $M_{*}$. Suppose we have proved that $M_{N}(t), M_{N-1}(t), \ldots, M_{r+1}(t) \rightarrow M_{*}$ as $t \rightarrow+\infty$ and have to show that $M_{r}(t) \rightarrow M_{*}$. Since $M_{r}(t) \leq M_{r+1}(t)$, it suffices to show that $\underset{t \rightarrow+\infty}{\lim } M_{r}(t) \geq$ $M_{*}$. Assume on the contrary that $\underset{t \rightarrow+\infty}{\lim } M_{r}(t)=m<M_{*}$. For any $\delta>0$, we have $M_{*}+\delta>M_{j}(t)>M_{*}-\delta$ for large $t>0$ and $j>r$. On the other hand, there exist a sequence $t_{n} \rightarrow \infty$, along which $M_{r}\left(t_{n}\right)<m+\delta$. Assume that $\delta>0$ is so small that $(m+\delta) \theta+\left(M_{*}+\delta\right)(1-\theta)<M_{*}-\delta$ and $(m+\delta) \theta_{0}+\left(M_{*}+\delta\right)\left(1-\theta_{0}\right)<M_{*}-\delta$, that is, $2 \delta<$ $\left(M_{*}-m\right) \max \left(\theta_{0}, \theta\right)$.

Accordingly to Lemma 15, applied for $t_{0}=t_{n}$ and $V=$ $\left\{k^{r+1}\left(t_{n}\right), \ldots, k^{N}\left(t_{n}\right)\right\}, M=M_{*}+\delta$ and $M^{\prime}=m_{*}+\delta$, there exists $j \in V$ such that $\left|x_{j}\left(t_{0}+T\right)\right|<M_{*}-\delta$. Similarly, for any $j \notin V$ we have $\left|x_{j}\left(t_{n}+T\right)\right|<M_{*}-\delta$ thanks to Lemma 14 Therefore, at time $t_{n}+T$ there are at least $r+1$ agents, whose opinions have moduli less than $M_{*}-\delta$ and hence $M_{r+1}\left(t_{n}+T\right) \leq M_{*}-\delta$ for any $n$. We arrived at the contradiction with the induction hypothesis. We thus proved that $M_{j}(t) \rightarrow M_{*} \forall j$ for any solution of (4), that is, modulus consensus is established. 
Proof of Corollary 1. By virtue of Theorem 2 modulus consensus is established. According to Lemma 1 it only three types of such a consensus are possible, which are stability, polarization and consensus. The common feature of the first two types is that for a.a. $x(0)$ there exists $i \in 1: N$ such that $\lim _{t \rightarrow+\infty} x_{i}(t) \leq 0$. It is well known [6], [9], [46] that the convex hull of the agents' states $\Delta(t)=\left[\min _{i} x_{i}(t), \max _{i} x_{i}(t)\right]$ is nonexpanding over time. Hence if $x_{i}(0) \geq 1 \forall i$, then $x_{i}(t) \geq 1$ for any $t \geq 0$, so the first two options are not possible.

\section{F. Proof of Lemma 6 and Theorems 5}

We start with proof of Lemma 6 , being a basis for Theorem 5 .

Proof of Lemma 6. We consider system (9), and the protocol (10) may be studied in the same way. Equation (11) is immediate from the definitions of $\mathfrak{a}_{i j}$ and $H_{i j}$. As follows from Lemma 3 , the solutions of (11) remain bounded since $|x(t)|_{\infty} \leq|x(0)|_{\infty}$. Since $H_{i j}(y, z)>0$ are continuous functions and the set $\left\{(y, z):|y|,|z| \leq|x(0)|_{\infty}\right\}$ is compact, there exist $M>m>0$ such that $m \leq H_{i j}[y, z] \leq M$ whenever $|y|,|z| \leq|x(0)|_{\infty}$. By substituting $y:=x_{j}(t) \operatorname{sgn} a_{i j}(t)$ and $z:=x_{i}(t)$, one shows that $m\left|a_{j k}\right| \leq\left|\mathfrak{a}_{j k}\right| \leq M\left|a_{j k}\right|$, from where the claim of Lemma 6 is obvious.

Now we proceed with the proofs of Theorems 5 and 6

Proof of Theorem 5. Since the right-hand sides of (9), (10) are smooth in $x$, the solutions exist locally and are unique. According to Lemma 3 and (11), the solutions remain bounded and thus infinitely prolongable. Under the USC assumption, modulus consensus follows from Theorem 2 and Lemma 6. If the graph $G[A(\cdot)]$ is ESC and cut-balanced, modulus consensus is implied by Theorem 3

Proof of Theorem 6. Using Lemma 3, one proves that the solution is bounded and hence its derivative also remains bounded due to [13, so any solution is infinitely prolongable. Since $x(t)$ is bounded, $A(t)$ is also bounded due to (13). The remaining claims follow now from Theorems 2,3

\section{CONCLUSIONS AND RELATED WORKS}

In the present paper, we extend a model of opinion dynamics in social networks with both attractive and repulsive interactions between the agents, which was proposed in recent papers by C. Altafini, who considered the conventional first-order consensus protocols over signed graphs. Altafini showed, in particular, the possibility of opinion polarization if the interaction graph is structurally balanced. In general, the protocol establishes modulus consensus, where the agents agree in modulus but may differ in signs (which not excludes convergence of all opinions to zero). In the present paper, we have examined dynamics of Altafini's protocols with switching directed topologies and offer sufficient conditions for reaching modulus consensus that boil down to uniform strong connectivity of the network. Moreover, under the assumption of cutbalance, the uniform connectivity may be further relaxed. In this case, we have obtained necessary and sufficient conditions for modulus consensus, classified into stability (converges of opinions to zero) and bipartite consensus (consensus or polarization). Getting rid of the restriction of static topologies allows to examine linear and nonlinear dynamics of social networks, where the agents may change their relations from friendship to hostility and vice versa. We are currently working with sociologists to test the theoretical results presented in this paper using data from human social groups.

\section{REFERENCES}

[1] W. Ren and R. Beard, Distributed consensus in multi-vehicle cooperative control: theory and applications. Springer, 2008.

[2] M. Mesbahi and M. Egerstedt, Graph Theoretic Methods in Multiagent Networks. Princeton and Oxford: Princeton University Press, 2010.

[3] W. Ren and Y. Cao, Distributed Coordination of Multi-agent Networks. Springer, 2011.

[4] M. DeGroot, "Reaching a consensus," Journal of the American Statistical Association, vol. 69, pp. 118-121, 1974.

[5] L. Moreau, "Stability of multiagent systems with time-dependent communication links," IEEE Trans. Autom. Control, vol. 50, no. 2, pp. 169$182,2005$.

[6] Z. Lin, B. Francis, and M. Maggiore, "State agreement for continuoustime coupled nonlinear systems," SIAM Journ. of Control and Optimization, vol. 46, no. 1, pp. 288-307, 2007.

[7] M. Cao, A. Morse, and B. Anderson, "Reaching a consensus in a dynamically changing environment: convergence rates, measurement delays, and asynchronous event," SIAM Journal of Control and Optimization, vol. 47, no. 2, pp. 601-623, 2008.

[8] V. Blondel, J. Hendrickx, A. Olshevsky, and J. Tsitsiklis, "Convergence in multiagent coordination, consensus, and flocking," in Proc. IEEE Conf. Decision and Control, 2005, pp. 2996 - 3000.

[9] A. Matveev, I. Novinitsyn, and A. Proskurnikov, "Stability of continuous-time consensus algorithms for switching networks with bidirectional interaction," in Proceedings of European Control Conference ECC-2013, 2013, pp. 1872-1877.

[10] J. Hendricx and J. Tsitsiklis, "Convergence of type-symmetric and cutbalanced consensus seeking systems," IEEE Trans. Autom. Control, vol. 58, no. 1, pp. 214-218, 2013.

[11] U. Münz, A. Papachristodoulou, and F. Allgöwer, "Consensus in multiagent systems with coupling delays and switching topology," IEEE Trans. Autom. Control, vol. 56, no. 12, pp. 2976-2982, 2011.

[12] G. Shi and K. Johansson, "Robust consensus for continuous-time multiagent dynamics," SIAM J. Control Optim, vol. 51, no. 5, pp. 3673-3691, 2013.

[13] — " "The role of persistent graphs in the agreement seeking of social networks," IEEE J. On Selected Areas In Communications, vol. 31, no. 9, pp. 595-606, 2013.

[14] L. Scardovi and R. Sepulchre, "Synchronization in networks of identical linear systems," Automatica, vol. 45, no. 11, pp. 2557-2562, 2009.

[15] J. Hu and W. Zheng, "Emergent collective behaviors on coopetition networks," Physica A, vol. 378, pp. 1787-1796, 2014.

[16] J. Hu and H. Zhu, "Adaptive bipartite consensus on coopetition networks," Physica D, vol. 307, pp. 14-21, 2015.

[17] D. Easley and J. Kleinberg, Networks, Crowds and Markets. Reasoning about a Highly Connected World. Cambridge: Cambridge Univ. Press, 2010.

[18] S. Wasserman and K. Faust, Social Network Analysis: Methods and Applications. Cambridge: Cambridge Univ. Press, 1994.

[19] A. Fläche and M. Macy, "Small worlds and cultural polarization," Journal of Math. Sociology, vol. 35, no. 1-3, pp. 146-176, 2011.

[20] J. Dillard and L. Shen, "On the nature of reactance and its role in persuasive health communication," Communication Monographs, vol. 72, no. 2, pp. 144-168, 2005.

[21] C. Hovland, I. Janis, and H. Kelley, Communication and persuasion. New Haven: Yale Univ. Press, 1953.

[22] E. Aronson, T. Wilson, and R. Akert, Social psychology. Upper Saddle River, NJ: Prentice Hall, 2010.

[23] W. Xia and M. Cao, "Clustering in diffusively coupled networks," Automatica, vol. 47, no. 11, pp. 2395-2405, 2011.

[24] R. Hegselmann and U. Krause, "Opinion dynamics and bounded confidence models, analysis, and simulation," Journal of Artifical Societies and Social Simulation (JASSS), vol. 5, no. 3, p. 2, 2002.

[25] G. Deffuant, D. Neau, F. Amblard, and G. Weisbuch, "Mixing beliefs among interacting agents," Advances in Complex Systems, vol. 3, pp. 87-98, 2000. 
[26] P. Dandekar, A. Goel, and D. Lee, "Biased assimilation, homophily, and the dynamics of polarization," PNAS, vol. 110, no. 15, pp. 5791-5796, 2013.

[27] C. Altafini, "Dynamics of opinion forming in structurally balanced social networks," PLOS ONE, vol. 7, no. 6, p. e38135, 2012.

[28] - "Consensus problems on networks with antagonistic interactions," IEEE Trans. Autom. Control, vol. 58, no. 4, pp. 935-946, 2013.

[29] Z. Meng, G. Shi, K. Johansson, M. Cao, and Y. Hong, "Modulus consensus over networks with antagonistic interactions and switching topologies," http://arxiv.org/abs/1402.2766, 2014.

[30] P. Romanczuk and L. Schimansky-Geier, "Swarming and pattern formation due to selective attraction and repulsion," Interface Focus, vol. 2, pp. 746-756, 2012.

[31] W. Yu, G. Chen, M. Cao, J. Lü, and H. Zhang, "Swarming behaviors in multi-agent systems with nonlinear dynamics," Chaos, vol. 23, p. 043118,2013

[32] C. Wang, G. Xie, and M. Cao, "Forming circle formations of anonymous mobile agents with order preservation," IEEE Trans. Autom. Control, vol. 58, no. 12, pp. 3248-3254, 2013.

[33] A. Zakhar'eva, A. S. Matveev, M. C. Hoy, and A. V. Savkin, "Distributed control of multiple non-holonomic robots with sector vision and range-only measurements for target capturing with collision avoidance," Robotica, vol. 33, pp. 385-412, 2015.

[34] K. Ovchinnikov, A. Semakova, and A. Matveev, "Decentralized multiagent tracking of unknown environmental level sets by a team of nonholonomic robots," in Proc. of 6th Int. Congress on Ultra Modern Communication and Control Systems (ICUMT), 2014, pp. 352-359.

[35] M. Valcher and P. Misra, "On the consensus and bipartite consensus in high-order multi-agent dynamical systems with antagonistic interactions," Systems Control Letters, vol. 66, pp. 94-103, 2014.

[36] H. Zhang and J. Chen, "Bipartite consensus of linear multi-agent systems over signed digraphs: An output feedback control approach," in Proc. of IFAC World Congress, 2014, pp. 2118 - 2123.

[37] J. Hendrickx, "A lifting approach to models of opinion dynamics with antagonisms," in Proc. of IEEE Conference on Decision and Control (CDC), 2014, pp. $2118-2123$

[38] C. Altafini and G. Lini, "Predictable dynamics of opinion forming for networks with antagonistic interactions," IEEE Trans. on Autom. Control, vol. 60, no. 2, pp. 342-357, 2015.

[39] W. Xia, M. Cao, and K. Johansson, "Structural balance and opinion separation in trust-mistrust social networks," IEEE Trans. on Control of Networks, 2015 (accepted).

[40] H. Smith, "Systems of ordinary differential equations which generate an order preserving flow. A survey of results." SIAM Review, vol. 30, pp. $87-113,1988$

[41] Z. Lin, B. Francis, and M. Maggiore, "Necessary and sufficient graphical conditions for formation control of unicycles," IEEE Trans. Autom. Control, vol. 50, no. 1, pp. 121-127, 2005.

[42] A. Proskurnikov and M. Cao, "Opinion dynamics using Altafini's model with a time-varying directed graph," in Proceedings of IEEE ISIC 2014 (Part of IEEE MSC 2014), Antibes, 2014, pp. 849-854.

[43] A. Proskurnikov, A. Matveev, and M. Cao, "Consensus and polarization in Altafini's model with bidirectional time-varying network topologies," in Proceedings of IEEE CDC 2014, Los Angeles, 2014, pp. 2112-2117.

[44] R. Olfati-Saber and R. Murray, "Consensus problems in networks of agents with switching topology and time-delays," IEEE Trans. Autom. Control, vol. 49, no. 9, pp. 1520-1533, 2004.

[45] P. Chebotarev and R. Agaev, "The forest consensus theorem," IEEE Trans. Autom. Control, vol. 59, no. 9, pp. 2475-2479, 2014

[46] L. Moreau, "Stability of continuous-time distributed consensus algorithms," in Proc. IEEE Conf. Decision and Control (CDC 2004), 2004, pp. $3998-4003$.

[47] S. Marvel, J. Kleinberg, R. Kleinberg, and S. Strogatz, "Continuous-time model of structural balance," PNAS, vol. 108, no. 5, pp. 1771-1776, 2011.

[48] T. Antal, P. Krapivsky, and S. Redner, "Dynamics of social balance on networks," Phys. Rev. E, vol. 72, p. 036121, 2005.

[49] R. Agaev and P. Chebotarev, "On the spectra of nonsymmetric laplacian matrices," Linear Algebra Appl., vol. 399, pp. 157-168, 2005.

[50] F. Clarke, "Generalized gradients and applications," Trans. of Amer. Math. Soc., vol. 205, pp. 247 - 262, 1975.

[51] E. McShane and R. Warfield, "On Filippov's implicit functions lemma," Proc. Amer. Math. Soc., vol. 18, pp. 41-47, 1967.

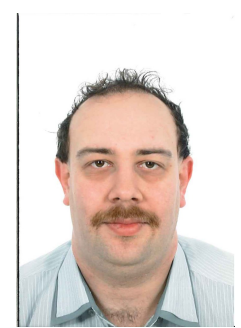

Anton V. Proskurnikov was born in Leningrad (now St. Petersburg) in 1982. He received his M.Sc. and Ph.D. degrees in 2003 and 2005 respectively, both from St.-Petersburg State University and supervised by Prof. V.A. Yakubovich. From 2003 till 2010 he was an Assistant Professor of the Department of Mathematics and Mechanics, St.Petersburg State University. Now A. Proskurnikov is a postdoctoral researcher at the ENTEG institute the University of Groningen, The Netherlands. He also occupies part-time researcher positions at St. Petersburg State University and Institute for Problems of Mechanical Engineering of Russian Academy of Sciences, St.Petersburg, Russia. His research interests include dynamics and control of complex networks, multi-agent and decentralized control nonlinear control, robust control, optimal control and optimization. In 2009 Anton Proskurnikov was awarded with a medal of Russian Academy of Sciences for prominent young researchers for a series of works on optimal tracking and disturbance rejection.

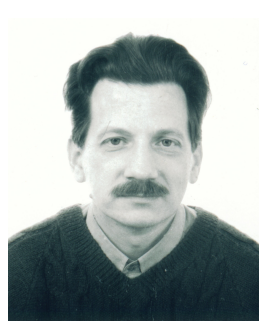

Alexey S. Matveev was born in Leningrad, USSR, in 1954. He received the M.S. and Ph.D. degrees in 1976 and 1980, respectively, both from the Leningrad University. Currently, he is a professor of the Department of Mathematics and Mechanics, St. Petersburg State University. His research interests include estimation and control over communication networks, hybrid dynamical systems, and navigation and control of mobile robots.

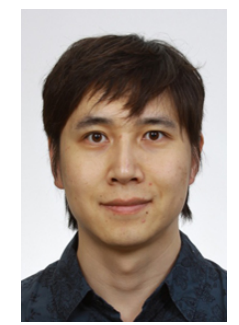

Ming Cao is currently a tenured associate professor responsible for the research direction of network analysis and control with the Faculty of Mathematics and Natural Sciences at the University of Groningen, the Netherlands, where he started as a tenure-track assistant professor in 2008. He received the Bachelor degree in 1999 and the Master degree in 2002 from Tsinghua University, Beijing, China, and the $\mathrm{PhD}$ degree in 2007 from Yale University, New Haven, CT, USA, all in electrical engineering. From September 2007 to August 2008, he was a postdoctoral research associate with the Department of Mechanical and Aerospace Engineering at Princeton University, Princeton, NJ, USA. He worked as a research intern during the summer of 2006 with the Mathematical Sciences Department at the IBM T. J. Watson Research Center, NY, USA His main research interest is in autonomous agents and multi-agent systems, mobile sensor networks and complex networks. He is an associate editor for Systems and Control Letters, and for the Conference Editorial Board of the IEEE Control Systems Society. He is also a member of the IFAC Technical Committee on Networked Systems. 